\title{
Vector relative degree and funnel control for differential-algebraic systems
}

\author{
Thomas Berger · Huy Hoàng Lê · Timo Reis
}

Received: date / Accepted: date

\begin{abstract}
We consider tracking control for multi-input multi-output differential-algebraic systems. First, the concept of vector relative degree is generalized for linear systems and we arrive at the novel concept of "truncated vector relative degree", and we derive a new normal form. Thereafter, we consider a class of nonlinear functional differential-algebraic systems which comprises linear systems with truncated vector relative degree. For this class we introduce a feedback controller which achieves that, for a given sufficiently smooth reference signal, the tracking error evolves within a prespecified performance funnel. We illustrate our results by an example of a robotic manipulator.
\end{abstract}

Keywords adaptive control $\cdot$ differential-algebraic equations $\cdot$ funnel control $\cdot$ relative degree

Mathematics Subject Classification (2010) 34A09 $\cdot 93 \mathrm{C} 05 \cdot 93 \mathrm{C} 10 \cdot 93 \mathrm{C} 23 \cdot 93 \mathrm{C} 40$

\section{Introduction}

Funnel control has been introduced in [22] almost two decades ago. Meanwhile, plenty of articles have been published in which funnel control from both a theoretical and an applied perspective are considered, see e.g. [3, 7, 10, 16, 17, 20, 25, 28] to mention only a few.

This work was supported by the German Research Foundation (Deutsche Forschungsgemeinschaft) via the grant BE 6263/1-1.

Corresponding author: Thomas Berger

Tel.: +495251 60-3779

Thomas Berger

Institut für Mathematik, Universität Paderborn, Warburger Str. 100, 33098 Paderborn, Germany

E-mail: thomas.berger@math.upb.de

Huy Hoàng Lê

Department of Mathematics, National University of Civil Engineering, 55 Giai Phong road, Dong Tam, Hai

Ba Trung, Hanoi, Vietnam

E-mail: hoanglh@nuce.edu.vn

Timo Reis

Fachbereich Mathematik, Universität Hamburg, Bundesstr. 55, 20146 Hamburg, Germany

E-mail: timo.reis@uni-hamburg.de 
A typical assumption in funnel control is that the system has a strict relative degree, which means that the input-output behavior can be described by a differential equation which has the same order for all outputs. However, multi-input, multi-output systems that appear in real-world applications do not always have a strict relative degree. Instead, the input-output behavior is described by a collection of differential equations of different order for each output, which is referred to as vector relative degree.

The subject of this article twofold: First we consider linear (not necessarily regular) systems described by differential-algebraic equations (DAEs). We generalize the notion of vector relative degree as given in [1, Def. 5.3.4] for regular DAEs, see [24 26] for systems of ordinary differential equations (ODEs). Furthermore, we develop a normal form for linear DAE systems which allows to read off this new truncated vector relative degree as well as the zero dynamics. Thereafter, we consider a class of nonlinear functional DAE systems which encompasses linear systems in this normal form, and we introduce a new funnel controller for this system class.

Our results generalize, on the one hand, the results of [7], where systems with strict relative degree are considered. On the other hand, concerning funnel control, the results in this article generalize those of [3,5] for linear and nonlinear DAEs, where the truncated vector relative degree (although this notion does not appear in these articles) is restricted to be component-wise less or equal to one. Note that [3] already encompasses the results found in [6] for linear DAE systems with properly invertible transfer function. DAEs with higher relative degree have been considered in [4], and even this article is comprised by the present results. Therefore, the present article can be seen as a unification of the funnel control results presented in the previous works [3-7] to a fairly general class of nonlinear DAE systems.

\subsection{Nomenclature}

Thoughout this article, $\mathbb{R}_{\geq 0}=[0, \infty)$ and $\|x\|$ is the Euclidean norm of $x \in \mathbb{R}^{n}$. The symbols $\mathbb{N}$ denotes the set of natural numbers and $\mathbb{N}_{0}=\mathbb{N} \cup\{0\}$. The ring of real polynomials is denoted by $\mathbb{R}[s]$, and $\mathbb{R}(s)$ is its quotient field. In other words, $\mathbb{R}(s)$ is the field of real rational functions. Further, $\mathbf{G l}_{n}(\mathbb{R})$ stands for the group of invertible matrices in $\mathbb{R}^{n \times n}$.

The restriction of a function $f: V \rightarrow \mathbb{R}^{n}$ to $W \subseteq V$ is denoted by $\left.f\right|_{W}, V \subseteq W$. For $p \in[1, \infty], L^{p}\left(I \rightarrow \mathbb{R}^{n}\right)\left(L_{\text {loc }}^{p}\left(I \rightarrow \mathbb{R}^{n}\right)\right)$ stands for the space of measurable and (locally) $p$-th power integrable functions $f: I \rightarrow \mathbb{R}^{n}, I \subseteq \mathbb{R}$ an interval. Likewise $L^{\infty}\left(I \rightarrow \mathbb{R}^{n}\right)\left(L_{\text {loc }}^{\infty}(I \rightarrow\right.$ $\left.\mathbb{R}^{n}\right)$ ) is the space of measurable and (locally) essentially bounded functions $f: I \rightarrow \mathbb{R}^{n}$, and $\|f\|_{\infty}$ stands for the essential supremum of $f$. Note that functions which agree almost everywhere are identified. Further, for $p \in[1, \infty]$ and $k \in \mathbb{N}_{0}, W^{k, p}\left(I \rightarrow \mathbb{R}^{n}\right)$ is the Sobolev space of elements of $L^{p}\left(I \rightarrow \mathbb{R}^{n}\right)\left(L_{\text {loc }}^{p}\left(I \rightarrow \mathbb{R}^{n}\right)\right)$ with the property that the first $k$ weak derivatives exist and are elements of $L^{p}\left(I \rightarrow \mathbb{R}^{n}\right)\left(L_{\text {loc }}^{p}\left(I \rightarrow \mathbb{R}^{n}\right)\right)$. Moreover, $C^{k}\left(V \rightarrow \mathbb{R}^{n}\right)$ is the set of $k$-times continuously differentiable functions $f: V \rightarrow \mathbb{R}^{n}, V \subseteq \mathbb{R}^{m}$, and we set $C\left(V \rightarrow \mathbb{R}^{n}\right):=C^{0}\left(V \rightarrow \mathbb{R}^{n}\right)$.

\section{Linear systems and the truncated vector relative degree}

In this section, we consider linear constant coefficient DAE systems

$$
\begin{aligned}
\frac{\mathrm{d}}{\mathrm{d} t} E x(t) & =A x(t)+B u(t), \\
y(t) & =C x(t),
\end{aligned}
$$


where $E, A \in \mathbb{R}^{l \times n}, B \in \mathbb{R}^{l \times m}, C \in \mathbb{R}^{p \times n}$. We denote the class of these systems by $\Sigma_{l, n, m, p}$ and write $[E, A, B, C] \in \Sigma_{l, n, m, p}$. We stress that these systems are not required to be regular, which would mean that $l=n$ and $\operatorname{det}(s E-A) \in \mathbb{R}[s] \backslash\{0\}$. The functions $u: \mathbb{R} \rightarrow \mathbb{R}^{m}$, $x: \mathbb{R} \rightarrow \mathbb{R}^{n}$, and $y: \mathbb{R} \rightarrow \mathbb{R}^{p}$ are called input, (generalized) state variable, and output of the system, respectively. We introduce the behavior of system (1) as

$$
\begin{aligned}
\mathfrak{B}_{[E, A, B, C]}:=\left\{(x, u, y) \in L_{\mathrm{loc}}^{1}\left(\mathbb{R} \rightarrow \mathbb{R}^{n} \times \mathbb{R}^{m} \times \mathbb{R}^{p}\right) \mid\right. \\
\left.E x \in W_{\mathrm{loc}}^{1,1}\left(\mathbb{R} \rightarrow \mathbb{R}^{l}\right) \wedge \frac{\mathrm{d}}{\mathrm{d} t} E x=A x+B u \wedge y=C x+D u\right\} .
\end{aligned}
$$

For a regular system $[E, A, B, C] \in \Sigma_{n, n, m, p}$, the transfer function is defined by

$$
G(s)=C(s E-A)^{-1} B \in \mathbb{R}(s)^{p \times m} .
$$

2.1 Zero dynamics and right-invertibility

To specify the class that we consider, we introduce the zero dynamics which are the set of solutions resulting in a trivial output. For more details on the concept of zero dynamics and a literature survey we refer to [1].

Definition 2.1. The zero dynamics of $[E, A, B, C] \in \Sigma_{l, n, m, p}$ are the set

$$
\mathscr{Z} \mathscr{D}_{[E, A, B, C]}:=\left\{(x, u, y) \in \mathfrak{B}_{[E, A, B, C]} \mid y=0\right\} .
$$

We call $\mathscr{Z} \mathscr{D}_{[E, A, B, C]}$ autonomous, if

$$
\forall \omega \in \mathscr{Z} \mathscr{D}_{[E, A, B, C]} \forall I \subseteq \mathbb{R} \text { open interval: }\left.\omega\right|_{I}=0 \Rightarrow \omega=0,
$$

and asymptotically stable, if

$$
\forall(x, u, y) \in \mathscr{Z} \mathscr{D}_{[E, A, B, C]}: \lim _{t \rightarrow \infty}\left\|\left.(x, u)\right|_{[t, \infty)}\right\|_{\infty}=0 .
$$

Remark 2.2. Let $[E, A, B, C] \in \Sigma_{l, n, m, p}$.

a) It has been shown in [3, Prop. 3.5] that

$$
\mathscr{Z} \mathscr{D}_{[E, A, B, C]} \text { are autonomous } \Longleftrightarrow \operatorname{ker}_{\mathbb{R}(s)}\left[\begin{array}{rr}
-s E+A & B \\
C & 0
\end{array}\right]=\{0\} .
$$

In particular, $\left[\begin{array}{rr}-s E+A & B \\ C & 0\end{array}\right]$ is left invertible over $\mathbb{R}(s)$ if, and only if, $\mathscr{Z} \mathscr{D}_{[E, A, B, C]}$ are autonomous. If $[E, A, B, C$ is regular, its transfer function $G(s)$ satisfies

$$
\left[\begin{array}{cr}
-s E+A & B \\
C & 0
\end{array}\right]\left[\begin{array}{cc}
I_{n} & (s E-A)^{-1} B \\
0 & I_{m}
\end{array}\right]=\left[\begin{array}{cc}
-s E+A & 0 \\
C & G(s)
\end{array}\right]
$$

hence autonomy of the zero dynamics is equivalent to $G(s)$ having full column rank over $\mathbb{R}(s)$, cf. [3, Prop. 4.8].

b) It has been shown in [3, Lem. 3.11] that

$$
\begin{aligned}
& \mathscr{Z} \mathscr{D}_{[E, A, B, C]} \text { are asymptotically stable } \\
\Longleftrightarrow & \operatorname{ker}_{\mathbb{C}}\left[\begin{array}{rr}
-\lambda E+A & B \\
C & 0
\end{array}\right]=\{0\} \text { for all } \lambda \in \mathbb{C}_{+} \text {with } \operatorname{Re}(\lambda) \geq 0 .
\end{aligned}
$$


We will consider systems with autonomous zero dynamics throughout this article. We will furthermore assume that the system is right-invertible, which is defined in the following.

Definition 2.3. The system $[E, A, B, C] \in \Sigma_{l, n, m, p}$ is called right-invertible, if

$$
\forall y \in C^{\infty}\left(\mathbb{R} \rightarrow \mathbb{R}^{p}\right) \exists(x, u) \in L_{\mathrm{loc}}^{1}\left(\mathbb{R} \rightarrow \mathbb{R}^{n} \times \mathbb{R}^{m}\right):(x, u, y) \in \mathfrak{B}_{[E, A, B, C]} .
$$

The notion of right-invertibility has been used in [29, Sec. 8.2] for systems governed by ordinary differential equations and in [2,3] for the differential-algebraic case. The concept is indeed motivated by tracking control: Namely, right-invertibility means that any smooth signal can be tracked by the output on a right-invertible system.

Remark 2.4. Consider a regular system $[E, A, B, C] \in \Sigma_{n, n, m, p}$ with transfer function $G(s)$. It has been shown in [3, Prop. 4.8] that

$$
[E, A, B, C] \text { is right-invertible } \Longleftrightarrow \operatorname{im}_{\mathbb{R}(s)} G(s)=\mathbb{R}(s)^{p},
$$

whence, by (2),

$$
[E, A, B, C] \text { is right-invertible } \Longleftrightarrow \operatorname{im}_{\mathbb{R}(s)}\left[\begin{array}{rr}
-s E+A & B \\
C & 0
\end{array}\right]=\mathbb{R}(s)^{n+p},
$$

Combining this with Remark 2.2 a) we can infer from the dimension formula that for regular square systems $[E, A, B, C] \in \Sigma_{n, n, m, m}$ (i.e., the dimensions of input and output coincide) with transfer function $G(s) \in \mathbb{R}(s)^{m \times m}$, the following statements are equivalent:

(i) $\mathscr{Z} \mathscr{D}_{[E, A, B, C]}$ autonomous,

(ii) $[E, A, B, C]$ is right-invertible,

(iii) $G(s) \in \mathbb{R}(s)^{m \times m}$ is invertible over $\mathbb{R}(s)$,

(iv) $\left[\begin{array}{rr}-s E+A & B \\ C & 0\end{array}\right]$ is invertible over $\mathbb{R}(s)$.

For general right-invertible systems with autonomous zero dynamics, we can derive a certain normal form under state space transformation. The following result is a straightforward combination of [3, Lem. 4.2 \& Thm. 4.3 \& Prop. 4.6].

Theorem 2.5. Let a right-invertible system $[E, A, B, C] \in \Sigma_{l, n, m, p}$ with autonomous zero $d y$ namics be given. Then there exist $W \in \mathbf{G l}_{l}(\mathbb{R}), T \in \mathbf{G l}_{n}(\mathbb{R})$ such that

$$
\begin{aligned}
W(s E-A) T & =\left[\begin{array}{ccc}
s I_{n_{1}}-Q & -A_{12} & 0 \\
-A_{21} & s E_{22}-A_{22} & s E_{23} \\
0 & s E_{32} & s N-I_{n_{3}} \\
0 & 0 & -s E_{43}
\end{array}\right], W B=\left[\begin{array}{c}
0 \\
I_{m} \\
0 \\
0
\end{array}\right], \\
C T & =\left[\begin{array}{lll}
0 & I_{p} & 0
\end{array}\right],
\end{aligned}
$$

where $n_{1}, n_{3}, n_{4} \in \mathbb{N}_{0}, N \in \mathbb{R}^{n_{3} \times n_{3}}$ is nilpotent and

$$
\begin{aligned}
Q \in \mathbb{R}^{n_{1} \times n_{1}}, \quad A_{12} & \in \mathbb{R}^{n_{1} \times p}, & A_{21} \in \mathbb{R}^{m \times n_{1}}, \\
E_{22}, A_{22} & \in \mathbb{R}^{m \times p}, & E_{23} \in \mathbb{R}^{m \times n_{3}}, \\
E_{32} & \in \mathbb{R}^{n_{3} \times p}, & E_{43} \in \mathbb{R}^{n_{4} \times n_{3}}
\end{aligned}
$$

are such that $E_{43} N^{j} E_{32}=0$ for all $j \in \mathbb{N}_{0}$. 
Remark 2.6. Let $[E, A, B, C] \in \Sigma_{l, n, m, p}$ be right-invertible and have autonomous zero dynamics. Using the form (3), we see that $(x, u, y) \in \mathfrak{B}_{[E, A, B, C]}$ if, and only if,

$$
T x=\left(\eta^{\top}, y^{\top}, x_{3}^{\top}\right)^{\top} \in L_{\mathrm{loc}}^{1}\left(\mathbb{R} \rightarrow \mathbb{R}^{n_{1}+p+n_{3}}\right)
$$

satisfies

$$
\left[\begin{array}{l}
E_{22} \\
E_{32}
\end{array}\right] y \in W_{\mathrm{loc}}^{1,1}\left(\mathbb{R} \rightarrow \mathbb{R}^{m+n_{3}}\right), \quad\left[\begin{array}{c}
E_{23} \\
N \\
E_{43}
\end{array}\right] x_{3} \in W_{\mathrm{loc}}^{1,1}\left(\mathbb{R} \rightarrow \mathbb{R}^{m+n_{3}+n_{4}}\right)
$$

and the equations

$$
\begin{aligned}
\dot{\eta} & =Q \eta+A_{12} y, \\
0 & =-\sum_{i=0}^{v-1} E_{23} N^{i} E_{32} y^{(i+2)}-E_{22} \dot{y}+A_{22} y+A_{21} \eta+u, \\
x_{3} & =\sum_{i=0}^{v-1} N^{i} E_{32} y^{(i+1)}
\end{aligned}
$$

holds in the distributional sense. In particular, the zero dynamics of $[E, A, B, C]$ are asymptotically stable if, and only if, any eigenvalue of $Q$ has negative real part.

Further note that $\eta \in L_{\text {loc }}^{1}\left(\mathbb{R} \rightarrow \mathbb{R}^{n_{1}}\right), y \in L_{\text {loc }}^{1}\left(\mathbb{R} \rightarrow \mathbb{R}^{p}\right)$ together with 5a imply that $\eta \in W_{\mathrm{loc}}^{1,1}\left(\mathbb{R} \rightarrow \mathbb{R}^{n_{1}}\right)$.

\subsection{Truncated vector relative degree}

Our aim in this section is to present a suitable generalization of the concept of vector relative degree to differential-algebraic systems which are not necessarily regular. For regular systems a definition of this concept is given in [3, Def. B.1].

Definition 2.7. Let a regular system $[E, A, B, C] \in \Sigma_{n, n, m, p}$ with transfer function $G(s) \in$ $\mathbb{R}(s)^{p \times m}$ be given. We say that $[E, A, B, C]$ has vector relative degree $\left(r_{1}, \ldots, r_{p}\right) \in \mathbb{Z}^{1 \times p}$, if there exists a matrix $\Gamma \in \mathbb{R}^{p \times m}$ with $\operatorname{rank} \Gamma=p$ and

$$
\lim _{\lambda \rightarrow \infty} \operatorname{diag}\left(\lambda^{r_{1}}, \ldots, \lambda^{r_{p}}\right) G(\lambda)=\Gamma .
$$

If the above holds with $r_{1}=\ldots=r_{p}=: r$, then we say that $[E, A, B, C]$ has strict relative degree $r$.

Since this definition involves the transfer function, it is only applicable to regular systems. To avoid this limitation, we introduce a novel concept. Let us start by introducing the notion of column degree of a rational matrix. This generalizes the concept of column degree for polynomial matrices in [15, Sec. 2.4].

Definition 2.8. For a rational function $r(s)=\frac{p(s)}{q(s)} \in \mathbb{R}(s)$ we define

$$
\operatorname{deg} r(s):=\operatorname{deg} p(s)-\operatorname{deg} q(s) .
$$

Further, for $r(s)=\left(r_{1}(s), r_{2}(s), \ldots, r_{p}(s)\right)^{\top} \in \mathbb{R}(s)^{p}$ we define

$$
\operatorname{deg} r(s)=\max _{1 \leq i \leq p} \operatorname{deg} r_{i}(s) .
$$


Note that the degree of a rational function $r(s)=\frac{p(s)}{q(s)}$ is independent of the choice of $p(s)$ and $q(s)$, i.e., they do not need to be coprime.

If $[E, A, B, C] \in \Sigma_{l, n, m, p}$ has autonomous zero dynamics, then we can conclude from Re$\operatorname{mark} 2.2$ that $\left[\begin{array}{rr}-s E+A & B \\ C & 0\end{array}\right] \in \mathbb{R}(s)^{(l+p) \times(n+m)}$ possesses a left inverse $L(s) \in \mathbb{R}(s)^{(n+m) \times(l+p)}$. Then we set

$$
H(s):=-\left[\begin{array}{ll}
0 & I_{m}
\end{array}\right] L(s)\left[\begin{array}{c}
0 \\
I_{p}
\end{array}\right] \in \mathbb{R}(s)^{m \times p} .
$$

\section{Remark 2.9.}

a) Assume that $[E, A, B, C] \in \Sigma_{l, n, m, p}$ has autonomous zero dynamics and is right-invertible. Then it has been shown in [3, Lem. A.1] that the rational matrix $H(s) \in \mathbb{R}(s)^{m \times p}$ is uniquely determined by $[E, A, B, C]$. Moreover, with the notation from Theorem 2.5 we have

$$
H(s)=s E_{22}-A_{22}-A_{21}\left(s I_{n_{1}}-Q\right)^{-1} A_{12}-s^{2} E_{23}\left(s N-I_{n_{3}}\right)^{-1} E_{32} .
$$

We stress that the above representation is independent of the transformation matrices $W$ and $T$ in (3).

b) If $[E, A, B, C] \in \Sigma_{n, n, m, m}$ has autonomous zero dynamics and is regular with transfer function $G(s) \in \mathbb{R}(s)^{m \times m}$, then, invoking (2) and Remark 2.4, it can be shown that $H(s)=G(s)^{-1}$, see also [3, Rem. A.4].

In view of Remark 2.9 we see that for any regular system $[E, A, B, C] \in \Sigma_{n, n, m, m}$ with transfer function $G(s)$ and vector relative degree $\left(r_{1}, \ldots, r_{m}\right)$, we have

$$
\begin{aligned}
& \lim _{\lambda \rightarrow \infty} \operatorname{diag}\left(\lambda^{r_{1}}, \ldots, \lambda^{r_{m}}\right) G(\lambda)=\Gamma \in \mathbf{G l}_{m}(\mathbb{R}) \\
\Longleftrightarrow & \lim _{\lambda \rightarrow \infty} H(\lambda) \operatorname{diag}\left(\lambda^{-r_{1}}, \ldots, \lambda^{-r_{m}}\right)=\Gamma^{-1} \in \mathbf{G l}_{m}(\mathbb{R}),
\end{aligned}
$$

with $H(s)$ as in (6). This motivates to use $H(s)$ instead of the transfer function $G(s)$ to define a generalization of the vector relative degree to DAE systems which are not necessarily regular.

Definition 2.10. Assume that $[E, A, B, C] \in \Sigma_{l, n, m, p}$ is right-invertible and has autonomous zero dynamics. Let $H(s) \in \mathbb{R}(s)^{m \times p}$ be defined as in (6) (which is well-defined by Re$\operatorname{mark} 2.9 \mathrm{a}], h_{i}(s)=H(s) e_{i} \in \mathbb{R}(s)^{m}$ for $i=1, \ldots, p$ and set $r_{i}=\max \left\{\operatorname{deg} h_{i}(s), 0\right\}$. Let $q$ be the number of nonzero entries of $\left(r_{1}, \ldots, r_{p}\right)$,

$$
\hat{\Gamma}:=\lim _{\lambda \rightarrow \infty} H(\lambda) \operatorname{diag}\left(\lambda^{-r_{1}}, \ldots, \lambda^{-r_{p}}\right) \in \mathbb{R}^{m \times p},
$$

and $\hat{\Gamma}_{q} \in \mathbb{R}^{m \times q}$ be the matrix which is obtained from $\hat{\Gamma}$ by deleting all the columns corresponding to $r_{i}=0$. Then we call $r=\left(r_{1}, \ldots, r_{p}\right) \in \mathbb{N}_{0}^{1 \times p}$ the truncated vector relative degree of the system $[E, A, B, C]$, if $\operatorname{rank} \hat{\Gamma}_{q}=q$.

A truncated vector relative degree $\left(r_{1}, \ldots, r_{p}\right)$ is called ordered, if $r_{1} \geq \ldots \geq r_{p}$.

Remark 2.11. Let the system $[E, A, B, C] \in \Sigma_{l, n, m, p}$ be right invertible and have autonomous zero dynamics.

a) Assume that $[E, A, B, C]$ has ordered truncated vector relative degree $\left(r_{1}, \ldots, r_{q}, 0, \ldots, 0\right)$ with $r_{q}>0$. Then the matrices $\hat{\Gamma}$ and $\hat{\Gamma}_{q}$ in Definition 2.10 are related by

$$
\hat{\Gamma}_{q}=\hat{\Gamma}\left[\begin{array}{c}
I_{q} \\
0
\end{array}\right]
$$


b) Assume that $[E, A, B, C]$ has truncated vector relative degree $\left(r_{1}, \ldots, r_{p}\right) \in \mathbb{N}_{0}^{1 \times p}$. Consider a permutation matrix $P_{\sigma} \in \mathbb{R}^{p \times p}$ induced by the permutation $\sigma:\{1, \ldots, p\} \rightarrow$ $\{1, \ldots, p\}$. A straightforward calculation shows that $H_{\sigma}(s)$ as in (6) corresponding to $\left[E, A, B, P_{\sigma} C\right]$ satisfies $H_{\sigma}(s)=H(s) P_{\sigma}$, thus the system $\left[E, A, B, P_{\sigma} C\right]$ has truncated vector relative degree $\left(r_{\sigma(1)}, \ldots, r_{\sigma(p)}\right)$. In particular, there exists a permutation $\sigma$ such that the output-permuted system $\left[E, A, B, P_{\sigma} C\right]$ has ordered truncated vector relative degree.

c) Assume that $[E, A, B, C]$ has ordered truncated vector relative degree $\left(r_{1}, \ldots, r_{p}\right) \in \mathbb{N}_{0}^{1 \times p}$. Using the notation from Theorem 2.5 and (7), we obtain that

$$
\begin{aligned}
& \hat{\Gamma}= \lim _{\lambda \rightarrow \infty} H(\lambda) \operatorname{diag}\left(\lambda^{-r_{1}}, \ldots, \lambda^{-r_{p}}\right) \\
&=\lim _{\lambda \rightarrow \infty}\left[\left(\lambda E_{22}-A_{22}\right)-A_{21}(\lambda I-Q)^{-1} A_{12}-\lambda^{2} E_{23}\left(\lambda N-I_{n_{3}}\right)^{-1} E_{32}\right] . \\
& \cdot \operatorname{diag}\left(\lambda^{-r_{1}}, \ldots, \lambda^{-r_{p}}\right) \\
&=\lim _{\lambda \rightarrow \infty}\left[\lambda E_{22}-A_{22}+\sum_{k=0}^{v-1} \lambda^{k+2} E_{23} N^{k} E_{32}\right] \operatorname{diag}\left(\lambda^{-r_{1}}, \ldots, \lambda^{-r_{p}}\right) .
\end{aligned}
$$

d) Consider a regular system $[E, A, B, C] \in \Sigma_{n, n, m, p}$. If $m>p$, then, in view of Remark 2.2 a), the zero dynamics of $[E, A, B, C]$ are not autonomous, because $\left[\begin{array}{cc}-s E+A & B \\ C & 0\end{array}\right]$ has a nontrivial kernel over $\mathbb{R}(s)$. Therefore, such a system does not have a truncated vector relative degree, but a vector relative degree may exist. As an example consider the system $[E, A, B, C] \in \Sigma_{1,1,2,1}$ with $E=C=[1], A=[0]$ and $B=[1,1]$, for which a truncated relative degree does not exist. However, the transfer function is given by $G(s)=s^{-1}[1,1]$ and hence the system even has strict relative degree $r=1$.

If $m \leq p$ and $[E, A, B, C]$ has a vector relative degree, then also a truncated vector relative degree exists. This can be seen as follows: First observe that, as a consequence of Definition 2.7 $p \leq m$ and hence we have $p=m$. Therefore, the matrix $\Gamma \in \mathbb{R}^{m \times m}$ in Definition 2.7 is invertible. Let $F(s):=\operatorname{diag}\left(\lambda^{r_{1}}, \ldots, \lambda^{r_{m}}\right) G(s)$, then $F(s)=\Gamma+G_{\mathrm{sp}}(s)$ for some strictly proper $G_{\mathrm{sp}}(s) \in \mathbb{R}(s)^{m \times m}$, i.e., $\lim _{\lambda \rightarrow \infty} G_{\mathrm{sp}}(\lambda)=0$. Then $\tilde{G}(s):=$ $-\Gamma^{-1} G_{\mathrm{sp}}(s)$ is strictly proper as well. Let $p(s) \in \mathbb{R}(s)^{m}$ be such that $F(s) p(s)=0$, then $p(s)=\tilde{G}(s) p(s)$. A component-wise comparison of the degrees yields that

$$
\forall i=1, \ldots, m: \operatorname{deg} p_{i}(s)=\operatorname{deg} \sum_{j=1}^{m} \tilde{G}_{i j}(s) p_{j}(s) \leq \max _{j=1, \ldots, m}\left(\operatorname{deg} p_{j}(s)-1\right),
$$

because $\operatorname{deg} \tilde{G}_{i j}(s) \leq-1$ for all $i, j=1, \ldots, m$. Therefore,

$$
\max _{i=1, \ldots, m} \operatorname{deg} p_{i}(s) \leq \max _{j=1, \ldots, m}\left(\operatorname{deg} p_{j}(s)-1\right)=\left(\max _{j=1, \ldots, m} \operatorname{deg} p_{j}(s)\right)-1,
$$

a contradiction. This shows that $F(s)$ is invertible over $\mathbb{R}(s)$ and hence $G(s)$ is invertible over $\mathbb{R}(s)$. Then Remark 2.4 yields that $[E, A, B, C]$ is right-invertible and has autonomous zero dynamics. Moreover, Remark 2.9 b) gives that $H(s)=-G(s)^{-1}$ and hence it follows that a truncated vector relative degree exists with $\hat{\Gamma}=-\Gamma^{-1}$ as in (9).

e) If $[E, A, B, C] \in \Sigma_{n, n, m, m}$ is regular and has autonomous zero dynamics, then $[E, A, B, C]$ has truncated vector relative degree $(0, \ldots, 0)$ if, any only if, the transfer function $G(s) \in$ $\mathbb{R}(s)^{m \times m}$ of $[E, A, B, C]$ is proper, i.e., $\lim _{\lambda \rightarrow \infty} G(\lambda) \in \mathbb{R}^{m \times m}$ exists. This is an immediate consequence of the fact that, by Remark 2.9 b), the matrix $H(s)$ in (6) satisfies $G(s)^{-1}$. 
f) A motivation for the definition of the truncated vector relative degree, even when only regular systems are considered, is given by output feedback control: Whilst the regular system $[E, A, B, C] \in \Sigma_{2,2,1,1}$ with

$$
E=\left[\begin{array}{ll}
0 & 1 \\
0 & 0
\end{array}\right], A=\left[\begin{array}{ll}
1 & 0 \\
0 & 1
\end{array}\right], B=\left[\begin{array}{l}
0 \\
1
\end{array}\right], C=\left[\begin{array}{ll}
1 & 0
\end{array}\right]
$$

has transfer function $G(s)=-s$ and thus vector relative degree $\left(r_{1}\right)=(-1)$, application of the static output feedback $u(t)=K y(t)+v(t)$ with new input $v$ leads to the system $[E, A+B K C, B, C]$ with transfer function $G_{K}(s)=\frac{-K}{1+K s}$. We may infer that the vector relative degree of $[E, A+B K C, B, C]$ is zero unless $K=0$, thus the vector relative degree is not invariant under output feedback in general.

In the following we show that the truncated vector relative degree is however invariant under static output feedback.

Proposition 2.12. Let $[E, A, B, C] \in \Sigma_{l, n, m, p}$ and $K \in \mathbb{R}^{m \times p}$ be given. Then the following statements hold:

a) $\mathscr{Z} \mathscr{D}_{[E, A, B, C]}$ are autonomous if, and only if, $\mathscr{Z}_{[E, A+B K C, B, C]}$ are autonomous.

b) $[E, A, B, C]$ is right-invertible if, and only if, $[E, A+B K C, B, C]$ is right-invertible.

c) $[E, A, B, C]$ has a truncated vector relative degree if, and only if, $[E, A+B K C, B, C]$ has a truncated vector relative degree. In this case, the truncated vector relative degrees of $[E, A, B, C]$ and $[E, A+B K C, B, C]$ coincide.

Proof. a) This follows from Remark 2.2 a) together with

$$
\left[\begin{array}{cr}
-s E+A+B K C & B \\
C & 0
\end{array}\right]=\left[\begin{array}{cc}
I_{l} & B K \\
0 & I_{p}
\end{array}\right]\left[\begin{array}{cc}
-s E+A & B \\
C & 0
\end{array}\right]
$$

b) Since $[E, A+B K C, B, C]$ is obtained from $[E, A, B, C]$ by output feedback $u(t)=K y(t)+$ $v(t)$ with new input $v \in L_{\mathrm{loc}}^{1}\left(\mathbb{R} \rightarrow \mathbb{R}^{m}\right)$, we obtain that $(x, u, y) \in \mathfrak{B}_{[E, A, B, C]}$ if, and only if, $(x, u-K y, y) \in \mathfrak{B}_{[E, A+B K C, B, C]}$. In particular, the set of generated outputs of $[E, A, B, C]$ and $[E, A+B K C, B, C]$ are the same, whence $[E, A, B, C]$ is right-invertible if, and only if, $[E, A+B K C, B, C]$ is right-invertible.

c) Since $[E, A, B, C]$ is obtained from $[E, A+B K C, B, C]$ by applying the feedback $-K$, it suffices to prove one implication. In view of Remark 2.11 b) it is no loss of generality to assume that $[E, A, B, C]$ has ordered truncated vector relative degree $\left(r_{1}, \ldots, r_{q}, 0 \ldots, 0\right) \in$ $\mathbb{N}_{0}^{1 \times p}$ with $r_{q}>0$. Let $L(s), L_{K}(s) \in \mathbb{R}(s)^{(n+m) \times(l+p)}$ be left inverses of

$$
\left[\begin{array}{cr}
-s E+A & B \\
C & 0
\end{array}\right] \text { and }\left[\begin{array}{cr}
-s E+A+B K C & B \\
C & 0
\end{array}\right], \quad \text { resp. }
$$

and partition

$$
L(s)=\left[\begin{array}{cc}
L_{11}(s) & L_{12}(s) \\
L_{21}(s) & H(s)
\end{array}\right]
$$

From (10) it follows that $L(s)\left[\begin{array}{cc}I_{l} & -B K \\ 0 & I_{p}\end{array}\right]$ is a left inverse of $\left[\begin{array}{c}-s E+A+B K C \\ C\end{array}\right]$. Since $H_{K}(s)=$ $\left[0, I_{m}\right] L_{K}(s)\left[\begin{array}{c}0 \\ I_{p}\end{array}\right]$ is independent of the choice of the left inverse $L_{K}(s)$ by Remark 2.9 a $)$, 
we may infer that

$$
\begin{aligned}
H_{K}(s) & =\left[\begin{array}{ll}
0 & I_{m}
\end{array}\right] L(s)\left[\begin{array}{cc}
I_{n} & -B K \\
0 & I_{m}
\end{array}\right]\left[\begin{array}{c}
0 \\
I_{p}
\end{array}\right] \\
& =\left[\begin{array}{ll}
0 & I_{m}
\end{array}\right]\left[\begin{array}{cc}
L_{11}(s) & L_{12}(s) \\
L_{21}(s) & H(s)
\end{array}\right]\left[\begin{array}{c}
-B K \\
I_{p}
\end{array}\right] \\
& =H(s)-L_{21}(s) B K .
\end{aligned}
$$

The relation $L(s)\left[\begin{array}{rr}-s E+A & B \\ C & 0\end{array}\right]=I_{n+m}$ leads to $L_{21}(s) B=I_{m}$. Therefore, $H_{K}(s)=H(s)-K$ and we find

$$
\begin{aligned}
\hat{\Gamma}_{K} & =\lim _{\lambda \rightarrow \infty} H_{K}(\lambda) \operatorname{diag}\left(\lambda^{-r_{1}}, \ldots, \lambda^{-r_{q}}, 1, \ldots, 1\right) \\
& =\hat{\Gamma}-\lim _{\lambda \rightarrow \infty} K \operatorname{diag}\left(\lambda^{-r_{1}}, \ldots, \lambda^{-r_{q}}, 1, \ldots, 1\right) .
\end{aligned}
$$

This implies that $\hat{\Gamma}_{K}\left[\begin{array}{c}I_{q} \\ 0\end{array}\right]=\hat{\Gamma}_{q}$, and thus

$$
\operatorname{rank} \hat{\Gamma}_{K}\left[\begin{array}{c}
I_{q} \\
0
\end{array}\right]=\operatorname{rank} \hat{\Gamma}_{q}=q
$$

Therefore, the truncated vector relative degree of the feedback system $[E, A+B K C, B, C]$ is $\left(r_{1}, \ldots, r_{q}, 0 \ldots, 0\right)$, i.e., that of $[E, A, B, C]$.

\section{Remark 2.13.}

a) The truncated vector relative degree of a right-invertible system with autonomous zero dynamics does not necessarily exist: For instance, consider $[E, A, B, C] \in \Sigma_{4,4,2,2}$ with

$$
E=\left[\begin{array}{llll}
1 & 0 & 0 & 0 \\
0 & 1 & 1 & 0 \\
0 & 1 & 1 & 0 \\
0 & 0 & 0 & 0
\end{array}\right], A=\left[\begin{array}{cccc}
-1 & 0 & 0 & 0 \\
0 & 1 & -1 & 0 \\
0 & 1 & 2 & 0 \\
0 & 0 & 0 & 1
\end{array}\right], B=\left[\begin{array}{ll}
0 & 0 \\
1 & 0 \\
0 & 1 \\
0 & 0
\end{array}\right], C=\left[\begin{array}{llll}
0 & 1 & 0 & 0 \\
0 & 0 & 1 & 0
\end{array}\right]
$$

For this system, we have

$$
H(s)=\left[\begin{array}{ll}
s-1 & s+1 \\
s-1 & s-2
\end{array}\right]
$$

Moreover,

$$
\hat{\Gamma}=\lim _{\lambda \rightarrow \infty} H(\lambda) \operatorname{diag}\left(\lambda^{-1}, \lambda^{-1}\right)=\left[\begin{array}{ll}
1 & 1 \\
1 & 1
\end{array}\right]=\hat{\Gamma}_{q} .
$$

Since $\operatorname{rank} \hat{\Gamma}_{q}=1<2$, which is the number of columns of $H(s)$ with positive degree. Hence, this system does not have a truncated vector relative degree.

b) There exist right-invertible regular systems with autonomous zero dynamics with the property that the truncated vector relative degree exists, but the vector relative degree according to Definition 2.7 does not exist. For instance, consider $[E, A, B, C] \in \Sigma_{5,5,2,2}$ with

$$
E=\left[\begin{array}{ccccc}
1 & 0 & 0 & 0 & 0 \\
0 & 1 & 0 & 1 & 0 \\
0 & -1 & 0 & 0 & 0 \\
0 & 0 & 0 & 0 & 1 \\
0 & 1 & 0 & 0 & 0
\end{array}\right], A=\left[\begin{array}{ccccc}
-1 & 1 & -2 & 0 & 0 \\
3 & 5 & 0 & 0 & 0 \\
0 & 0 & 0 & 0 & 0 \\
0 & 0 & 0 & 1 & 0 \\
0 & 0 & 0 & 0 & 1
\end{array}\right], B=\left[\begin{array}{ll}
0 & 0 \\
1 & 0 \\
0 & 1 \\
0 & 0 \\
0 & 0
\end{array}\right], C=\left[\begin{array}{lllll}
0 & 1 & 0 & 0 & 0 \\
0 & 0 & 1 & 0 & 0
\end{array}\right]
$$


Then

We have

$$
G(s)=C(s E-A)^{-1} B=\left[\begin{array}{cc}
0 & -\frac{1}{s} \\
\frac{s+1}{6} & \frac{s^{4}+s^{3}+s^{2}-4 s-8}{6 s}
\end{array}\right] .
$$

$$
\Gamma:=\lim _{\lambda \rightarrow \infty} \operatorname{diag}\left(\lambda, \lambda^{-3}\right) G(\lambda)=\left[\begin{array}{cc}
0 & -1 \\
0 & \frac{1}{6}
\end{array}\right], \text { and } \operatorname{rank} \Gamma=1<2 .
$$

This implies that the system does not have vector relative degree in the sense of Definition 2.7 Invoking Remark 2.9 b) we obtain

$$
H(s)=G(s)^{-1}=\left[\begin{array}{cc}
\frac{s^{4}+s^{3}+s^{2}-4 s-8}{s+1} & \frac{6}{s+1} \\
-s & 0
\end{array}\right],
$$

and

$$
\hat{\Gamma}:=\lim _{\lambda \rightarrow \infty} H(\lambda) \operatorname{diag}\left(\lambda^{-3}, 1\right)=\left[\begin{array}{ll}
1 & 0 \\
0 & 0
\end{array}\right] \text { and } \hat{\Gamma}_{q}=\left[\begin{array}{l}
1 \\
0
\end{array}\right] \text {. }
$$

Then $\operatorname{rank} \hat{\Gamma}_{q}=1=q$, and consequently this system has truncated vector relative degree $(3,0)$.

\subsection{A representation for systems with truncated vector relative degree}

For ODE systems, BYRNES and ISIDORI have introduced a normal form under state space transformation which allows to read off the relative degree and internal dynamics [11, 24]. This normal form plays an important role in designing local and global stabilizing feedback controllers for nonlinear systems [12]-14], adaptive observers [27], and adaptive controllers [19, 23]. A normal form for linear ODE systems with vector relative degree has been developed in [26]. Further, a normal form for regular linear DAE systems with strict relative degree has been derived in [4], whereas a normal form for regular linear differentialalgebraic systems with proper inverse transfer function in [6]. The latter has been extended to (not necessarily regular) DAE systems with truncated vector relative degree pointwise less or equal to one in [3], although this notion was not used there. Note that the concept of truncated vector relative degree encompasses systems governed by ODEs with strict or vector relative degree as well as regular DAE systems with strict relative degree (up to some extent, cf. Remark 2.11 d) or proper inverse transfer function, and we introduce a novel representation which comprises all the aforementioned results.

Assume that $[E, A, B, C] \in \Sigma_{l, n, m, p}$ is right-invertible, has autonomous zero dynamics and has possesses a truncated vector relative degree $\left(r_{1}, \ldots, r_{p}\right) \in \mathbb{N}_{0}^{1 \times p}$. By Remark $2.11 \mathrm{~b}$ ) it is further no loss of generality to assume that the latter is ordered, i.e., $r_{1} \geq \ldots \geq r_{q}>0=$ $r_{q+1}=\ldots=r_{p}$. Introduce the polynomial matrix

$$
F(s):=s E_{22}-A_{22}+\sum_{k=0}^{v-1} s^{k+2} E_{23} N^{k} E_{32} \in \mathbb{R}(s)^{m \times p} .
$$

By Remark 2.11 c) we have

$$
\begin{aligned}
\hat{\Gamma} & =\lim _{\lambda \rightarrow \infty} H(\lambda) \operatorname{diag}\left(\lambda^{-r_{1}}, \ldots, \lambda^{-r_{q}}, 1, \ldots, 1\right)=\lim _{\lambda \rightarrow \infty} F(\lambda) \operatorname{diag}\left(\lambda^{-r_{1}}, \ldots, \lambda^{-r_{q}}, 1, \ldots, 1\right) \\
& =\left[\begin{array}{ll}
\hat{\Gamma}_{11} & \hat{\Gamma}_{12} \\
\hat{\Gamma}_{21} & \hat{\Gamma}_{22}
\end{array}\right] \in \mathbb{R}^{m \times p},
\end{aligned}
$$


where the latter partition is with $\hat{\Gamma}_{11} \in \mathbb{R}^{q \times q}, \hat{\Gamma}_{12} \in \mathbb{R}^{q \times(p-q)}, \hat{\Gamma}_{21} \in \mathbb{R}^{(m-q) \times q}$ and $\hat{\Gamma}_{22} \in$ $\mathbb{R}^{(m-q) \times(p-q)}$. Then Definition 2.10 yields

$$
\operatorname{rank}\left[\begin{array}{c}
\hat{\Gamma}_{11} \\
\hat{\Gamma}_{21}
\end{array}\right]=\operatorname{rank} \hat{\Gamma}\left[\begin{array}{c}
I_{q} \\
0
\end{array}\right]=q
$$

Let $h \in \mathbb{N}$ be such that $r_{h}>1$ and $r_{h+1}=1$. Denote the $j$ th column of a matrix $M$ by $M^{(j)}$. Then

$$
\begin{aligned}
\hat{\Gamma} & =\lim _{\lambda \rightarrow \infty} F(\lambda) \operatorname{diag}\left(\lambda^{-r_{1}}, \ldots, \lambda^{-r_{q}}, 1, \ldots, 1\right) \\
& =\left[\begin{array}{llllll}
E_{23} N^{r_{1}-2} E_{32}^{(1)} & \ldots E_{23} N^{r_{h}-2} E_{32}^{(h)} & E_{22}^{(h+1)} & \ldots E_{22}^{(q)} & -A_{22}^{(q+1)} & \ldots-A_{22}^{(p)}
\end{array}\right],
\end{aligned}
$$

and thus

$$
\hat{\Gamma}_{q}=\left[\begin{array}{l}
\hat{\Gamma}_{11} \\
\hat{\Gamma}_{21}
\end{array}\right]=\left[\begin{array}{llllll}
E_{23} N^{r_{1}-2} E_{32}^{(1)} & \ldots & E_{23} N^{r_{h}-2} E_{32}^{(h)} & E_{22}^{(h+1)} & \ldots & E_{22}^{(q)}
\end{array}\right] \in \mathbb{R}^{m \times q} .
$$

Since $\operatorname{rank} \hat{\Gamma}_{q}=q$, by reordering the inputs and - accordingly - reording the rows of $A_{21}$, $E_{22}, A_{22}$ and $E_{23}$, it is no loss of generality to assume that the first $q$ rows of $\hat{\Gamma}_{q}$ are linearly independent, thus $\hat{\Gamma}_{11} \in \mathbf{G I}_{q}(\mathbb{R})$. Consider the matrix

$$
\Gamma:=\left[\begin{array}{cc}
\Gamma_{11} & 0 \\
\Gamma_{21} & I_{m-q}
\end{array}\right] \in \mathbf{G l}_{m}(\mathbb{R}),
$$

where $\Gamma_{11}=\hat{\Gamma}_{11}^{-1} \in \mathbf{G l}_{q}(\mathbb{R}), \Gamma_{21}=-\hat{\Gamma}_{21} \hat{\Gamma}_{11}^{-1} \in \mathbb{R}^{(m-q) \times q}$, then

$$
\Gamma \hat{\Gamma}_{q}=\left[\begin{array}{c}
I_{q} \\
0
\end{array}\right] .
$$

On the other hand, using the notation from Theorem 2.5 and invoking Remark 2.6 we have that $(x, u, y) \in \mathfrak{B}_{[E, A, B, C]}$ if, and only if, $T x=\left(\eta^{\top}, y^{\top}, x_{3}^{\top}\right)^{\top} \in L_{\mathrm{loc}}^{1}\left(\mathbb{R} \rightarrow \mathbb{R}^{n_{1}+p+n_{3}}\right)$ solves (5) in the distributional sense, and the components satisfy (4). Since (5b) can be written as $F\left(\frac{\mathrm{d}}{\mathrm{d} t}\right) y=A_{21} \eta+u$, by construction of $\hat{\Gamma}_{q}$ and (12) we may rewrite this as

$$
\hat{\Gamma}_{q}\left(\begin{array}{c}
y_{1}^{\left(r_{1}\right)} \\
\vdots \\
y_{q}^{\left(r_{q}\right)}
\end{array}\right)=M_{1}\left(\begin{array}{c}
y_{1} \\
\vdots \\
y_{1}^{\left(r_{1}-1\right)}
\end{array}\right)+\ldots+M_{q}\left(\begin{array}{c}
y_{q} \\
\vdots \\
y_{q}^{\left(r_{q}-1\right)}
\end{array}\right)+M\left(\begin{array}{c}
y_{q+1} \\
\vdots \\
y_{m}
\end{array}\right)+A_{21} \eta+u
$$

for some $M_{1} \in \mathbb{R}^{m \times r_{1}}, \ldots, M_{q} \in \mathbb{R}^{m \times r_{q}}, M \in \mathbb{R}^{m \times(p-q)}$ which can be constructed from the columns of $E_{23} N^{i} E_{32}, E_{22}$ and $A_{22}, i=0, \ldots, r_{1}$. Define $R_{j, 1} \in \mathbb{R}^{q \times r_{j}}, R_{j, 2} \in \mathbb{R}^{(m-q) \times r_{j}}$ for $j=1, \ldots, q$ and $S_{1} \in \mathbb{R}^{q \times(p-q)}, S_{2} \in \mathbb{R}^{(m-q) \times(p-q)}, P_{1} \in \mathbb{R}^{q \times n_{1}}, P_{2} \in \mathbb{R}^{(m-q) \times n_{1}}$ by

$$
\left[\begin{array}{l}
R_{j, 1} \\
R_{j, 2}
\end{array}\right]:=\Gamma M_{j}, \quad j=1, \ldots, q, \quad\left[\begin{array}{l}
S_{1} \\
S_{2}
\end{array}\right]:=\Gamma M, \quad\left[\begin{array}{l}
P_{1} \\
P_{2}
\end{array}\right]:=\Gamma A_{21}
$$


By a multiplication of (15) from the left with $\Gamma \in \mathbf{G l}_{m}(\mathbb{R})$, we obtain that, also invoking 5a and $5 \mathrm{c}$,

$$
\begin{aligned}
\dot{\eta}= & Q \eta+A_{12} y \\
\left(\begin{array}{c}
y_{1}^{\left(r_{1}\right)} \\
\vdots \\
y_{q}^{\left(r_{q}\right)}
\end{array}\right)= & R_{1,1}\left(\begin{array}{c}
y_{1} \\
\vdots \\
y_{1}^{\left(r_{1}-1\right)}
\end{array}\right)+\ldots+R_{q, 1}\left(\begin{array}{c}
y_{q} \\
\vdots \\
y_{q}^{\left(r_{q}-1\right)}
\end{array}\right)+S_{1}\left(\begin{array}{c}
y_{q+1} \\
\vdots \\
y_{m}
\end{array}\right)+P_{1} \eta+\Gamma_{11}\left(\begin{array}{c}
u_{1} \\
\vdots \\
u_{q}
\end{array}\right), \\
0= & R_{1,2}\left(\begin{array}{c}
y_{1} \\
\vdots \\
y_{1}^{\left(r_{1}-1\right)}
\end{array}\right)+\ldots+R_{q, 2}\left(\begin{array}{c}
y_{q} \\
\vdots \\
y_{q}^{\left(r_{q}-1\right)}
\end{array}\right)+S_{2}\left(\begin{array}{c}
y_{q+1} \\
\vdots \\
y_{m}
\end{array}\right) \\
& +P_{2} \eta+\Gamma_{21}\left(\begin{array}{c}
u_{1} \\
\vdots \\
u_{q}
\end{array}\right)+\left(\begin{array}{c}
u_{q+1} \\
\vdots \\
u_{m}
\end{array}\right) \\
x_{3}= & \sum_{i=0}^{v-1} N^{i} E_{32} y^{(i+1)} .
\end{aligned}
$$

We have thus derived a representation for systems with truncated vector relative degree and summarize the findings in the following result.

Theorem 2.14. Let a right-invertible system $[E, A, B, C] \in \Sigma_{l, n, m, p}$ with autonomous zero dynamics be given. Assume that $[E, A, B, C]$ has ordered truncated vector relative degree $\left(r_{1}, \ldots, r_{q}, 0, \ldots, 0\right)$ with $r_{q}>0$. Use the notation from Theorem 2.5, (13) and (16). Then $(x, u, y) \in \mathfrak{B}_{[E, A, B, C]}$, if, and only if, after a reordering of the inputs so that $\hat{\Gamma}_{11}$ in (12) is invertible,

$$
T x=\left(\eta^{\top}, y^{\top}, x_{3}^{\top}\right)^{\top} \in L_{\mathrm{loc}}^{1}\left(\mathbb{R} \rightarrow \mathbb{R}^{n_{1}+p+n_{3}}\right)
$$

satisfies the smoothness conditions in (4) and solves (17) in the distributional sense.

Remark 2.15. Consider a regular and right-invertible system $[E, A, B, C] \in \Sigma_{n, n, m, m}$ with autonomous zero dynamics and ordered truncated vector relative degree $\left(r_{1}, \ldots, r_{q}, 0, \ldots, 0\right) \in$ $\mathbb{N}_{0}^{1 \times p}$ such that $r_{q}>0$.

a) If $[E, A, B, C]$ has strict relative degree $r>0$, then $q=m$ and $r_{1}=\ldots=r_{m}=r$. In this case, the representation (17) simplifies to

$$
\begin{aligned}
\dot{\eta} & =Q \eta+A_{12} y \\
y^{(r)} & =R_{1,1}\left(\begin{array}{c}
y_{1} \\
\vdots \\
y_{1}^{(r-1)}
\end{array}\right)+\ldots+R_{m, 1}\left(\begin{array}{c}
y_{m} \\
\vdots \\
y_{m}^{(r-1)}
\end{array}\right)+P_{1} \eta+\Gamma_{11} u \\
x_{3} & =\sum_{i=0}^{v-1} N^{i} E_{32} y^{(i+1)}
\end{aligned}
$$

Since the second equation can be rewritten as

$$
y^{(r)}=Q_{r-1} y^{(r-1)}+\ldots+Q_{0} y+P_{1} \eta+\Gamma_{11} u
$$

for matrices $Q_{0}, \ldots, Q_{r-1}$, this is exactly the form which has been developed in [4]. 
b) If the transfer function $G(s) \in \mathbb{R}(s)^{m \times m}$ of $[E, A, B, C]$ has a proper inverse, then we have that $H(s)=G(s)^{-1}$ (see Remark 2.9 b) is proper, hence $q=0$ and the truncated vector relative degree is $(0, \ldots, 0)$. In this case, the representation (17) simplifies to

$$
\begin{aligned}
\dot{\eta} & =Q \eta+A_{12} y, \\
0 & =S_{2} y+P_{2} \eta+u, \\
x_{3} & =\sum_{i=0}^{v-1} N^{i} E_{32} y^{(i+1)},
\end{aligned}
$$

which is exactly the form developed in [6].

c) If the system is an ODE, that is $E=I_{n}$, then its transfer function $G(s)$ is strictly proper, i.e., $\lim _{\lambda \rightarrow \infty} G(\lambda)=0$. We can further infer from Remark 2.4 that the transfer function $G(s) \in \mathbb{R}(s)^{m \times m}$ is invertible. Then (8) implies $q=m$, i.e., the truncated vector relative degree (which coincides with the vector relative degree by Remark 2.11d) is $\left(r_{1}, \ldots, r_{m}\right) \in \mathbb{N}^{1 \times m}$. In this case, (17) simplifies to

$$
\begin{aligned}
\dot{\eta} & =Q \eta+A_{12} y \\
\left(\begin{array}{c}
y_{1}^{\left(r_{1}\right)} \\
\vdots \\
y_{m}^{\left(r_{m}\right)}
\end{array}\right) & =R_{1,1}\left(\begin{array}{c}
y_{1} \\
\vdots \\
y_{1}^{\left(r_{1}-1\right)}
\end{array}\right)+\ldots+R_{m, 1}\left(\begin{array}{c}
y_{m} \\
\vdots \\
y_{m}^{\left(r_{m}-1\right)}
\end{array}\right)+P_{1} \eta+\Gamma_{11} u \\
x_{3} & =\sum_{i=0}^{v-1} N^{i} E_{32} y^{(i+1)}
\end{aligned}
$$

This form comprises the one presented in [26], where, additionally,

$$
\begin{aligned}
x_{3} & =\left(\dot{y}_{1}, \ldots, y_{1}^{\left(r_{1}-1\right)}, \ldots, \dot{y}_{m}, \ldots, y_{m}^{\left(r_{m}-1\right)}\right)^{\top} \in \mathbb{R}^{n_{3}}, \\
N & =\operatorname{diag}\left(N_{1}, \ldots, N_{m}\right) \in \mathbb{R}^{n_{3} \times n_{3}} \text { with } N_{i}=\left[{ }^{0} \prod_{0}\right] \in \mathbb{R}^{\left(r_{i}-1\right) \times\left(r_{i}-1\right)}, \\
E_{32} & =\operatorname{diag}\left(e_{1}^{\left[r_{1}-1\right]}, \ldots, e_{1}^{\left[r_{1}-1\right]}\right) \in \mathbb{R}^{n_{3} \times m}
\end{aligned}
$$

where $e_{1}^{[k]} \in \mathbb{R}^{k}$ is the first canonical unit vector. We note that the above nilpotent matrix $N$ has index $v=\max _{1 \leq i \leq m}\left(r_{i}-1\right)$.

\section{Nonlinear systems with truncated vector relative degree}

In this section, we consider a class of nonlinear DAE systems which comprises the class of linear DAE systems which have a truncated vector relative degree and the same number of inputs and outputs. More precisely, we consider nonlinear functional differential-algebraic 
systems of the form

$$
\begin{aligned}
\left(\begin{array}{c}
y_{1}^{\left(r_{1}\right)}(t) \\
y_{2}^{\left(r_{2}\right)}(t) \\
\vdots \\
y_{q}^{\left(r_{q}\right)}(t)
\end{array}\right)= & f_{1}\left(d_{1}(t), T_{1}\left(y_{1}, \ldots, y_{1}^{\left(r_{1}-1\right)}, \ldots, y_{q}^{\left(r_{q}-1\right)}, y_{q+1}, \ldots, y_{m}\right)(t)\right) \\
& +\Gamma_{I}\left(d_{2}(t), T_{1}\left(y_{1}, \ldots, y_{1}^{\left(r_{1}-1\right)}, \ldots, y_{q}^{\left(r_{q}-1\right)}, y_{q+1}, \ldots, y_{m}\right)(t)\right) u_{I}(t), \\
0= & f_{2}\left(y_{1}(t), \ldots, y_{1}^{\left(r_{1}-1\right)}(t), \ldots, y_{q}^{\left(r_{q}-1\right)}(t), y_{q+1}(t), \ldots, y_{m}(t)\right) \\
& +f_{3}\left(d_{3}(t),\left(T_{2} y\right)(t)\right)+\Gamma_{I I}\left(d_{4}(t),\left(T_{2} y\right)(t)\right) u_{I}(t)+f_{4}\left(d_{5}(t),\left(T_{2} y\right)(t)\right) u_{I I}(t), \\
\left.y\right|_{[-h, 0]}= & y^{0}
\end{aligned}
$$

with initial data

$$
\begin{aligned}
y^{0}=\left(y_{1}^{0}, y_{2}^{0}, \ldots, y_{m}^{0}\right)^{\top}, \quad y_{i}^{0} \in C^{r_{i}-1}([-h, 0] \rightarrow \mathbb{R}), \quad i=1, \ldots, q, \\
y_{i}^{0} \in C([-h, 0] \rightarrow \mathbb{R}), \quad i=q+1, \ldots, m,
\end{aligned}
$$

where $f_{1}, \ldots, f_{4}, \Gamma_{I}, \Gamma_{I I}, d_{1}, \ldots, d_{5}$ are functions and $T_{1}, T_{2}$ are operators with properties being specified in the sequel. The output is $y=\left(y_{1}, \ldots, y_{m}\right)^{\top}$ and the input of the system is $u=\left(u_{1}, \ldots, u_{m}\right)^{\top}$, for which we set

$$
u_{I}=\left(u_{1}, \ldots, u_{q}\right)^{\top}, u_{I I}=\left(u_{q+1}, \ldots, u_{m}\right)^{\top},
$$

i.e., $u=\left(u_{I}^{\top}, u_{I I}^{\top}\right)^{\top}$. The functions $d_{1}, \ldots, d_{5}: \mathbb{R}_{\geq 0} \rightarrow \mathbb{R}^{s}$ play the roles of disturbances. We denote $\bar{r}=r_{1}+\ldots+r_{p}$ and call - in virtue of Section 2.3 - the tuple $\left(r_{1}, \ldots, r_{p}, 0, \ldots, 0\right) \in$ $\mathbb{N}_{0}^{1 \times m}$ with $r_{i}>0$ for $i=1, \ldots, q$ the truncated vector relative degree of (18). We will later show that linear DAE systems which have a truncated vector relative degree belong to this class. Similar to [5], we introduce the following classes of operators.

Definition 3.1. For $m, k \in \mathbb{N}$ and $h \geq 0$ the set $\mathbb{T}_{m, k, h}$ denotes the class of operators $T$ : $C\left([-h, \infty) \rightarrow \mathbb{R}^{m}\right) \rightarrow L_{\text {loc }}^{\infty}\left(\mathbb{R}_{\geq 0} \rightarrow \mathbb{R}^{k}\right)$ with the following properties:

(i) $T$ is causal, i.e, for all $t \geq 0$ and all $\zeta, \xi \in C\left([-h, \infty) \rightarrow \mathbb{R}^{m}\right)$,

$$
\left.\zeta\right|_{[-h, t)}=\left.\left.\xi\right|_{[-h, t)} \Longrightarrow T(\zeta)\right|_{[0, t)}=\left.T(\xi)\right|_{[0, t)} .
$$

(ii) $T$ is locally Lipschitz continuous in the following sense: for all $t \geq 0$ and all $\xi \in$ $C\left([-h, t] \rightarrow \mathbb{R}^{m}\right)$ there exist $\tau, \delta, c>0$ such that, for all $\zeta_{1}, \zeta_{2} \in \mathscr{C}\left([-h, \infty) \rightarrow \mathbb{R}^{m}\right)$ with $\left.\zeta_{i}\right|_{[-h, t]}=\xi$ and $\left\|\zeta_{i}(s)-\xi(t)\right\|<\delta$ for all $s \in[t, t+\tau]$ and $i=1,2$, we have

$$
\left\|\left.\left(T\left(\zeta_{1}\right)-T\left(\zeta_{2}\right)\right)\right|_{[t, t+\tau]}\right\|_{\infty} \leq c\left\|\left.\left(\zeta_{1}-\zeta_{2}\right)\right|_{[t, t+\tau]}\right\|_{\infty} .
$$

(iii) $T$ maps bounded trajectories to bounded trajectories, i.e, for all $c_{1}>0$, there exists $c_{2}>0$ such that for all $\zeta \in \mathscr{C}\left([-h, \infty) \rightarrow \mathbb{R}^{m}\right)$

$$
\left\|\left.\zeta\right|_{[-h, \infty)}\right\|_{\infty} \leq c_{1} \Longrightarrow\left\|\left.T(\zeta)\right|_{[0, \infty)}\right\|_{\infty} \leq c_{2} .
$$

Furthermore, the set $\mathbb{T}_{m, k, h}^{\mathrm{DAE}}$ denotes the subclass of operators

$$
T: C\left([-h, \infty) \rightarrow \mathbb{R}^{m}\right) \rightarrow C^{1}\left(\mathbb{R}_{\geq 0} \rightarrow \mathbb{R}^{k}\right)
$$

such that $T \in \mathbb{T}_{m, k, h}$ and, additionally, 
(iv) there exist $z \in C\left(\mathbb{R}^{m} \times \mathbb{R}^{k} \rightarrow \mathbb{R}^{k}\right)$ and $\tilde{T} \in \mathbb{T}_{m, k, h}$ such that

$$
\forall \zeta \in C\left([-h, \infty) \rightarrow \mathbb{R}^{m}\right) \forall t \geq 0: \frac{\mathrm{d}}{\mathrm{d} t}(T \zeta)(t)=z(\zeta(t),(\tilde{T} \zeta)(t)) .
$$

Assumption 3.2. We assume that the functional differential-algebraic system (18) has the following properties:

(i) the gain $\Gamma_{I} \in C\left(\mathbb{R}^{s} \times \mathbb{R}^{k} \rightarrow \mathbb{R}^{q \times q}\right)$ satisfies $\Gamma_{I}(d, \eta)+\Gamma_{I}(d, \eta)^{\top}>0$ for all $(d, \eta) \in$ $\mathbb{R}^{s} \times \mathbb{R}^{k}$, and $\Gamma_{I I} \in C^{1}\left(\mathbb{R}^{s} \times \mathbb{R}^{k} \rightarrow \mathbb{R}^{(m-q) \times q}\right)$.

(ii) the disturbances satisfy $d_{1}, d_{2} \in L^{\infty}\left(\mathbb{R}_{\geq 0} \rightarrow \mathbb{R}^{s}\right)$ and $d_{3}, d_{4}, d_{5} \in W^{1, \infty}\left(\mathbb{R}_{\geq 0} \rightarrow \mathbb{R}^{s}\right)$.

(iii) $f_{1} \in C\left(\mathbb{R}^{s} \times \mathbb{R}^{k} \rightarrow \mathbb{R}^{q}\right), f_{2} \in C^{1}\left(\mathbb{R}^{\bar{r}+m-q} \rightarrow \mathbb{R}^{m-q}\right), f_{3} \in C^{1}\left(\mathbb{R}^{s} \times \mathbb{R}^{k} \rightarrow \mathbb{R}^{m-q}\right)$, and $f_{2}^{\prime}\left[\begin{array}{c}0 \\ I_{m-q}\end{array}\right]$ is bounded.

(iv) $f_{4} \in C^{1}\left(\mathbb{R}^{s} \times \mathbb{R}^{k} \rightarrow \mathbb{R}\right)$ and there exists $\alpha>0$ such that $f_{4}(d, v) \geq \alpha$ for all $(d, v) \in$ $\mathbb{R}^{s} \times \mathbb{R}^{k}$

(v) $T_{1} \in \mathbb{T}_{\bar{r}+m-q, k, h}$ and $T_{2} \in \mathbb{T}_{m, k, h}^{\mathrm{DAE}}$.

In the remainder of this section we show that any right-invertible system $[E, A, B, C] \in$ $\Sigma_{l, n, m, m}$ with truncated vector relative degree $\left(r_{1}, \ldots, r_{q}, 0, \ldots, 0\right)$, where $r_{1}, \ldots, r_{q} \in \mathbb{N}$, belongs to the class of systems (18) which satisfy Assumption 3.2 as long as $[E, A, B, C]$ has asymptotically stable zero dynamics and the matrix $\Gamma_{11}$ in 17 satisfies $\Gamma_{11}+\Gamma_{11}^{\top}>0$. We have seen in Remark 2.6 that asymptotic stability of the zero dynamics is equivalent to the matrix $Q$ in 17 having only eigenvalues with negative real part.

Consider the three first equations in 17) and the operator

$$
\begin{aligned}
T_{2}: \quad C\left([0, \infty) \rightarrow \mathbb{R}^{m}\right) & \rightarrow C^{1}\left(\mathbb{R}_{\geq 0} \rightarrow \mathbb{R}^{n_{3}}\right) \\
y & \mapsto\left(t \mapsto\left(T_{2} y\right)(t):=\eta(t)=e^{Q t} \eta^{0}+\int_{0}^{t} e^{Q(t-\tau)} A_{12} y(\tau) d \tau\right),
\end{aligned}
$$

which is parameterized by the initial value $\eta^{0} \in \mathbb{R}^{n_{1}}$. This operator is clearly causal, locally Lipschitz, and, since all eigenvalues of $Q$ have negative real part, $T$ satisfies property (iii) in Definition 3.1 The derivative is given by

$$
\frac{\mathrm{d}}{\mathrm{d} t}\left(T_{2} y\right)(t)=Q e^{Q t} \eta^{0}+A_{12} y(t)+Q \int_{0}^{t} e^{Q(t-\tau)} A_{12} y(\tau) d \tau=:(\tilde{T} y)(t), \quad t \geq 0,
$$

and it is straightforward to check that $\tilde{T} \in \mathbb{T}_{m, n_{3}, 0}$. Therefore, we obtain that $T_{2} \in \mathbb{T}_{m, n_{3}, 0}^{\mathrm{DAE}}$. Further consider the operator $T_{1}: C\left([0, \infty) \rightarrow \mathbb{R}^{\bar{r}+m-q}\right) \rightarrow L_{\text {loc }}^{\infty}\left(\mathbb{R}_{\geq 0} \rightarrow \mathbb{R}^{q}\right)$ defined by

$$
\begin{aligned}
& T_{1}\left(\zeta_{1,1}, \ldots, \zeta_{1, r_{1}}, \ldots, \zeta_{q, r_{q}}, \zeta_{q+1,1}, \ldots, \zeta_{m, 1}\right) \\
& =R_{1,1}\left(\begin{array}{c}
\zeta_{1,1} \\
\vdots \\
\zeta_{1, r_{1}}
\end{array}\right)+\ldots+R_{q, 1}\left(\begin{array}{c}
\zeta_{q, 1} \\
\vdots \\
\zeta_{q, r_{q}}
\end{array}\right)+S_{1}\left(\begin{array}{c}
\zeta_{q+1,1} \\
\vdots \\
\zeta_{m, 1}
\end{array}\right)+P_{1} T_{2}\left(\zeta_{1,1}, \zeta_{2,1}, \ldots, \zeta_{m, 1}\right),
\end{aligned}
$$

then, likewise, we obtain that $T_{1} \in \mathbb{T}_{\bar{r}+m-q, q, 0}$. The remaining functions are given by

$$
f_{1}(d, \eta)=\eta, \Gamma_{I}(d, \eta)=\Gamma_{11}, f_{3}(d, \eta)=P_{2} \eta, \Gamma_{I I}(d, \eta)=\Gamma_{21}, f_{4}(d, \eta)=1
$$


and

$$
\begin{aligned}
& f_{2}\left(\zeta_{1,1}, \ldots, \zeta_{1, r_{1}}, \ldots, \zeta_{q, r_{q}}, \zeta_{q+1,1}, \ldots, \zeta_{m, 1}\right) \\
& =R_{1,2}\left(\begin{array}{c}
\zeta_{1,1} \\
\vdots \\
\zeta_{1, r_{1}}
\end{array}\right)+\ldots+R_{q, 2}\left(\begin{array}{c}
\zeta_{q, 1} \\
\vdots \\
\zeta_{q, r_{q}}
\end{array}\right)+S_{2}\left(\begin{array}{c}
\zeta_{q+1,1} \\
\vdots \\
\zeta_{m, 1}
\end{array}\right)
\end{aligned}
$$

The function $f_{2}$ satisfies condition (iii) in Assumption 3.2 since

$$
f_{2}^{\prime}\left[\begin{array}{c}
0 \\
I_{m-q}
\end{array}\right]=S_{2} \in \mathbb{R}^{(m-q) \times(m-q)} .
$$

Note that system (17) does not entirely belong to the class (18) since the fourth equation in 177 is not included. However, the control objective formulated in the following section can also be achieved for (17), see also Remark 4.1 e)

\section{Funnel control}

\subsection{Control objective}

Let a reference signal $y_{\mathrm{ref}}=\left(y_{\mathrm{ref}, 1}, \ldots, y_{\mathrm{ref}, m}\right)^{\top}$ with $y_{\mathrm{ref}, i} \in W^{r_{i}, \infty}\left(\mathbb{R}_{\geq 0} \rightarrow \mathbb{R}\right)$ for $i=1, \ldots, q$ and $y_{\mathrm{ref}, i} \in W^{1, \infty}\left(\mathbb{R}_{\geq 0} \rightarrow \mathbb{R}\right)$ for $i=q+1, \ldots, m$ be given, and let $e=y-y_{\mathrm{ref}}$ be the tracking error. The objective is to design an output error feedback of the form

$$
u(t)=F\left(t, e_{1}(t), \ldots, e_{1}^{\left(r_{1}-1\right)}(t), \ldots, e_{q}^{\left(r_{q}-1\right)}(t), e_{q+1}(t), \ldots, e_{m}(t)\right)
$$

such that in the closed-loop system the tracking error evolves within a prescribed performance funnel

$$
\mathscr{F}_{\varphi}^{m}:=\left\{(t, e) \in \mathbb{R}_{\geq 0} \times \mathbb{R}^{m} \mid \varphi(t)\|e\|<1\right\},
$$

which is determined by a function $\varphi$ belonging to

$$
\Phi_{k}:=\left\{\begin{array}{l|l}
\varphi \in C^{k}\left(\mathbb{R}_{\geq 0} \rightarrow \mathbb{R}\right) & \begin{array}{l}
\varphi, \dot{\varphi}, \ldots, \varphi^{(k)} \text { are bounded, } \\
\varphi(\tau)>0 \text { for all } \tau>0, \text { and } \liminf _{\tau \rightarrow \infty} \varphi(\tau)>0
\end{array}
\end{array}\right\}
$$

A further objective is that all signals $u, e_{1}, \ldots, e_{1}^{\left(r_{1}-1\right)}, \ldots, e_{q}^{\left(r_{q}-1\right)}, e_{q+1}, \ldots, e_{m}: \mathbb{R}_{\geq 0} \rightarrow \mathbb{R}^{m}$ should remain bounded.

The funnel boundary is given by the reciprocal of $\varphi$, see Fig. 1 It is explicitly allowed that $\varphi(0)=0$, meaning that no restriction on the initial value is imposed since $\varphi(0)\|e(0)\|<1$; the funnel boundary $1 / \varphi$ has a pole at $t=0$ in this case. Since every $\varphi \in \Phi_{k}$ is bounded, the boundary of the associated performance funnel $\mathscr{F}_{\varphi}^{m}$ is bounded away from zero, which means that there exists $\lambda>0$ with $1 / \varphi(t) \geq \lambda$ for all $t>0$. Further note that the funnel boundary is not necessarily monotonically decreasing, but it might be beneficial to choose a wider funnel over some later time interval, for instance in the presence of periodic disturbance or when the reference signal varias strongly. Various different funnel boundaries are possible, see e.g. [18, Sec. 3.2]. 


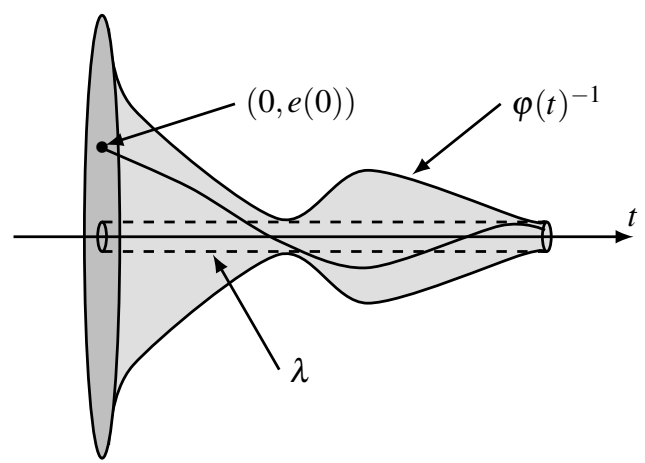

Fig. 1: Error evolution in a funnel $\mathscr{F}_{\varphi}^{1}$ with boundary $\varphi(t)^{-1}$ for $t>0$.

\subsection{Controller design}

The funnel controller for systems of the form (18) satisfying Assumption 3.2 is of the following form:

$$
\begin{aligned}
& \text { For } i=1, \ldots, q \text { : } \\
& e_{i 0}(t)=e_{i}(t)=y_{i}(t)-y_{\mathrm{ref}, i}(t), \\
& e_{i 1}(t)=\dot{e}_{i 0}(t)+k_{i 0}(t) e_{i 0}(t), \\
& e_{i 2}(t)=\dot{e}_{i 1}(t)+k_{i 1}(t) e_{i 1}(t), \\
& \text { : } \\
& e_{i, r_{i}-1}(t)=\dot{e}_{i, r_{i}-2}(t)+k_{i, r_{i}-2}(t) e_{i, r_{i}-2}(t), \\
& k_{i j}(t)=\frac{1}{1-\varphi_{i j}^{2}(t)\left|e_{i j}(t)\right|^{2}}, \quad j=0, \ldots, r_{i}-2 . \\
& \text { For } i=q+1, \ldots, m: e_{i}(t)=y_{i}(t)-y_{\mathrm{ref}, i}(t) \text {, } \\
& e_{I}(t)=\left(e_{1, r_{1}-1}(t), \ldots, e_{q, r_{q}-1}(t)\right)^{\top}, \quad e_{I I}(t)=\left(e_{q+1}(t), \ldots, e_{m}(t)\right)^{\top}, \\
& k_{I}(t)=\frac{1}{1-\varphi_{I}(t)^{2}\left\|e_{I}(t)\right\|^{2}}, \quad k_{I I}(t)=\frac{\hat{k}}{1-\varphi_{I I}(t)^{2}\left\|e_{I I}(t)\right\|^{2}}, \\
& u(t)=\left(\begin{array}{c}
u_{I}(t) \\
u_{I I}(t)
\end{array}\right)=\left(\begin{array}{c}
-k_{I}(t) e_{I}(t) \\
-k_{I I}(t) e_{I I}(t)
\end{array}\right)
\end{aligned}
$$

where we impose the following conditions on the reference signal and funnel functions:

$$
\begin{gathered}
y_{\mathrm{ref}}=\left(y_{\mathrm{ref}, 1}, \ldots, y_{\mathrm{ref}, m}\right)^{\top}, \quad y_{\mathrm{ref}, i} \in W^{r_{i}, \infty}\left(\mathbb{R}_{\geq 0} \rightarrow \mathbb{R}\right), i=1, \ldots, q \\
y_{\mathrm{ref}, i} \in W^{1, \infty}\left(\mathbb{R}_{\geq 0} \rightarrow \mathbb{R}\right), i=q+1, \ldots, m \\
\varphi_{I}, \varphi_{I I} \in \Phi_{1}, \varphi_{i j} \in \Phi_{r_{i}-j}, i=1, \ldots, q, j=0, \ldots, r_{i}-2 .
\end{gathered}
$$

We further assume that $\hat{k}$ satisfies

$$
\hat{k}>\alpha^{-1} \sup _{Y \in \mathbb{R}^{\Gamma+m-q}}\left\|f_{2}^{\prime}(Y)\left[\begin{array}{c}
0 \\
I_{m-q}
\end{array}\right]\right\| .
$$




\section{Remark 4.1.}

a) By a solution of the closed-loop system (18), (22) on $[-h, \omega), \omega \in(0, \infty]$, with initial data $y^{0}$ as in 190 we mean a function $y=\left(y_{1}, \ldots, y_{m}\right)^{\top}$ such that $\left.y\right|_{[-h, 0]}=y^{0}$, $y_{i} \in C^{r_{i}-1}([-h, \omega) \rightarrow \mathbb{R})$ and $\left.y_{i}^{\left(r_{i}-1\right)}\right|_{[0, \omega)}$ is weakly differentiable for $i=1, \ldots, q, y_{i} \in$ $C([-h, \omega] \rightarrow \mathbb{R})$ and $\left.y_{i}\right|_{[0, \omega)}$ is weakly differentiable for $i=q+1, \ldots, m$, and $y$ satisfies the differential-algebraic equation in (18) with $u$ defined in (22) in the weak sense. The solution $y$ is called maximal, if it has no right extension that is also a solution, and global, if $\omega=\infty$.

b) Assumption 3.2 (iii) together with condition (24) are essential for the solvability of the closed-loop system (18), 22), since they guarantee the invertibility of $\alpha \hat{k} I_{m-q}-$ $f_{3}^{\prime}(Y)\left[\begin{array}{c}0 \\ I_{m-q}\end{array}\right]$. This property is crucial for the explicit solution of the algebraic constraint in the closed-loop system (18), 22).

c) If the system (18) has strict relative, i.e., $q=m$ and $r_{1}=\ldots=r_{m}=: r>0$, then it satisfies the assumptions of [7, Thm. 3.1]. In this case, the funnel controller (22) simplifies to

$$
\begin{aligned}
& \text { For } i=1, \ldots, m, \\
& \qquad \begin{aligned}
e_{i 0}(t) & =e_{i}(t)=y_{i}(t)-y_{\mathrm{ref}, i}(t), \\
e_{i 1}(t) & =\dot{e}_{i 0}(t)+k_{i 0}(t) e_{i 0}(t), \\
e_{i 2}(t) & =\dot{e}_{i 1}(t)+k_{i 1}(t) e_{i 1}(t), \\
& \vdots \\
e_{i, r-1}(t) & =\dot{e}_{i, r-2}(t)+k_{i, r-2}(t) e_{i, r-2}(t), \\
k_{i j}(t) & =\frac{1}{1-\varphi_{i j}^{2}(t)\left|e_{i j}(t)\right|^{2}}, \quad j=0, \ldots, r-2, \\
e_{r-1}(t) & =\left(e_{1, r-1}(t), \ldots, e_{m, r-1}(t)\right)^{\top} \\
k_{r-1}(t) & =\frac{1}{1-\varphi_{r-1}(t)^{2}\left\|e_{r-1}(t)\right\|^{2}} \\
u(t) & =-k_{r-1}(t) e_{r-1}(t)
\end{aligned}
\end{aligned}
$$

This controller slightly differs from the one presented in [7] for systems with strict relative degree (even when we choose $\varphi_{i j}=\varphi_{1 j}$ for all $i=1, \ldots, m$ ), which reads

$$
\begin{aligned}
e_{0}(t) & =e(t)=y(t)-y_{\mathrm{ref}}(t) \\
e_{1}(t) & =\dot{e}_{0}(t)+k_{0}(t) e_{0}(t) \\
e_{2}(t) & =\dot{e}_{1}(t)+k_{1}(t) e_{1}(t) \\
& \vdots \\
e_{r-1}(t) & =\dot{e}_{r-2}(t) k_{r-2}(t) e_{r-2}(t) \\
k_{i}(t) & =\frac{1}{1-\varphi_{i}(t)^{2}\left\|e_{i}(t)\right\|^{2}}, \quad i=0, \ldots, r-1 \\
u(t) & =-k_{r-1}(t) e_{r-1}(t)
\end{aligned}
$$

d) If the system (18) satisfies $q=0$, then the funnel controller (22) simplifies to

$$
\begin{aligned}
& e(t)=y(t)-y_{\mathrm{ref}}(t), \quad k(t)=\frac{\hat{k}}{1-\varphi(t)^{2}\|e(t)\|^{2}}, \\
& u(t)=-k(t) e(t),
\end{aligned}
$$

and feasibility follows from the results in [5] where funnel control for this type has been considered. 
e) Let us stress again that a linear system of the form (17) does not completely belong to the class (18) as the fourth equation in (17) is not included. However, we like to emphasize that in

$$
x_{3}(t)=\sum_{i=0}^{v-1} N^{i} E_{32} y^{(i+1)}(t),
$$

the output $y$ is required smooth enough for $x_{3}$ to be well defined. Nevertheless, the funnel controller (22) can also be applied to systems of the form (17). To see this, assume that there exists a solution to (22) applied to (17) except for the fourth equation. If the funnel functions $\varphi_{I}, \varphi_{I I}$ and $\varphi_{i j}, i=1, \ldots, q, j=0, \ldots, r_{i}-2$ are additionally in $C^{v+1}\left(\mathbb{R}_{\geq 0} \rightarrow\right.$ $\mathbb{R})$ and $y_{\text {ref }}$ is additionally in $W^{v+2, \infty}\left(\mathbb{R}_{\geq 0} \rightarrow \mathbb{R}^{m}\right)$, then the solution $\left.y\right|_{[0, \infty)}$ will be at least in $C^{v+1}\left(\mathbb{R}_{>0} \rightarrow \mathbb{R}^{m}\right)$, so that $x_{3}$ is well defined and continuously differentiable. The proof of this statement is similar to Step 2 of the proof of [3, Thm. 5.3]. Furthermore, using $y_{\text {ref }} \in W^{v+2, \infty}\left(\mathbb{R}_{\geq 0} \rightarrow \mathbb{R}^{m}\right)$ also yields boundedness of $x_{3}$, cf. Step 4 of the proof of [3, Thm. 5.3].

Remark 4.2. Consider a system (18) which satisfies Assumption 3.2 and let the reference signal and funnel functions be as in (23). Since the second equation in (18) is an algebraic equation we need to guarantee that it is initially satisfied for a solution to exist. Since $T_{2} \in$ $\mathbb{T}_{m, k, h}^{\mathrm{DAE}}$ is causal it "localizes", in a natural way, to an operator $\hat{T}_{2}: C\left([-h, \omega] \rightarrow \mathbb{R}^{n}\right) \rightarrow$ $C^{1}\left([0, \omega] \rightarrow \mathbb{R}^{k}\right)$, cf. [21, Rem. 2.2]. With some abuse of notation, we will henceforth not distinguish between $T_{2}$ and its "localization" $\hat{T}_{2}$. Note that for $\omega=0$ we have that $\hat{T}_{2}$ : $C\left([-h, 0] \rightarrow \mathbb{R}^{n}\right) \rightarrow \mathbb{R}^{k}$. Hence, an initial value $y^{0}$ as in $[19]$ is called consistent for the closed loop system (18), 22], if

$$
\begin{aligned}
& f_{2}\left(y_{1}^{0}(0), \ldots,\left(\frac{\mathrm{d}}{\mathrm{d} t}\right)^{r_{1}-1}\left(y_{1}^{0}\right)(0), \ldots,\left(\frac{\mathrm{d}}{\mathrm{d} t}\right)^{r_{q}-1}\left(y_{q}^{0}\right)(0), y_{q+1}^{0}(0), \ldots, y_{m}^{0}(0)\right) \\
& +f_{3}\left(d_{3}(0), T_{2}\left(y^{0}\right)\right)+\Gamma_{I I}\left(d_{4}(0), T_{2}\left(y^{0}\right)\right) u_{I}(0)+f_{4}\left(d_{5}(0), T_{2}\left(y^{0}\right)\right) u_{I I}(0)=0,
\end{aligned}
$$

where $u_{I}(0), u_{I I}(0)$ are defined by (22).

\subsection{Feasibility of funnel control}

We show feasibility of the funnel controller (22) for systems of the form (18) satisfying Assumption 3.2 The following theorem unifies and extends the funnel control results from [3], which are all special cases of it.

Theorem 4.3. Consider a system (18) satisfying Assumption 3.2 Let $y_{\mathrm{ref}}$ and $\varphi_{I}, \varphi_{I I}, \varphi_{i j}$, $i=1, \ldots, q, j=0, \ldots, r_{i}-2$ be as in (23) and $\hat{k}>0$ such that (24) holds. Then for any consistent initial value $y^{0}$ as in (19) (i.e., $y^{0}$ satisfies (26) such that $e_{I}, e_{I I}, e_{i j}, i=1, \ldots, q$, $j=0, \ldots, r_{i}-2$ defined in 22, satisfy

$$
\begin{aligned}
& \varphi_{I}(0)\left\|e_{I}(0)\right\|<1, \quad \varphi_{I I}(0)\left\|e_{I I}(0)\right\|<1, \\
& \varphi_{i j}(0)\left|e_{i j}(0)\right|<1, \quad i=1, \ldots, q, j=0, \ldots, r_{i}-2,
\end{aligned}
$$

the application of the funnel controller (22) to (18) yields a closed-loop initial value problem that has a solution and every solution can be extended to a global solution. Furthermore, for every global solution $y(\cdot)$,

(i) the input $u: \mathbb{R}_{\geq 0} \rightarrow \mathbb{R}^{m}$ and the gain functions $k_{I}, k_{I I}, k_{i j}: \mathbb{R}_{\geq 0} \rightarrow \mathbb{R}, i=1, \ldots, q, j=$ $0, \ldots, r_{i}-2$ are bounded; 
(ii) the functions $e_{I}: \mathbb{R}_{\geq 0} \rightarrow \mathbb{R}^{q}, e_{I I}: \mathbb{R}_{\geq 0} \rightarrow \mathbb{R}^{m-q}$ and $e_{i j}: \mathbb{R}_{\geq 0} \rightarrow \mathbb{R}, i=1, \ldots, q, j=$ $0, \ldots, r_{i}-2$ evolve in their respective performance funnels, i.e., for all $i=1, \ldots, q, j=$ $0, \ldots, r_{i}-2$ and $t \geq 0$ we have

$$
\left(t, e_{I}(t)\right) \in \mathscr{F}_{\varphi_{I}}^{q},\left(t, e_{I I}(t)\right) \in \mathscr{F}_{\varphi_{I I}}^{m-q},\left(t, e_{i j}(t)\right) \in \mathscr{F}_{\varphi_{i j}}^{1}
$$

Furthermore, the signals $e_{I}(\cdot), e_{I I}(\cdot), e_{i j}(\cdot)$ are uniformly bounded away from the funnel boundaries in the following sense:

$$
\begin{array}{lr}
\exists \varepsilon_{I}>0 \forall t>0: & \left\|e_{I}(t)\right\| \leq \varphi_{I}(t)^{-1}-\varepsilon_{I}, \\
\exists \varepsilon_{I I}>0 \forall t>0: & \left\|e_{I I}(t)\right\| \leq \varphi_{I I}(t)^{-1}-\varepsilon_{I I}, \\
\forall i=1, \ldots, q, j=0, \ldots, r_{i}-2 \exists \varepsilon_{i j}>0 \forall t>0: & \left|e_{i j}(t)\right| \leq \varphi_{i j}(t)^{-1}-\varepsilon_{i j} .
\end{array}
$$

In particular, each error component $e_{i}(t)=y_{i}-y_{\mathrm{ref}, i}(t)$ evolves in the funnel $\mathscr{F}_{\varphi_{i 0}}^{1}$, for $i=1, \ldots, q$, or $\mathscr{F}_{\varphi_{I I}}^{1}$, for $i=q+1, \ldots, m$, resp., and stays uniformly away from its boundary.

The proof of this theorem is similar to the one of [7, Thm. 3.1], where the feasibility of the funnel controller (25) for ODE systems with strict relative degree has been treated. However, one of the additional difficulties in proving this theorem is that the closed-loop system (18), 22) is now a DAE because of the second equation in (18).

Proof. We proceed in several steps.

Step 1: We show that a maximal solution $y:[-h, \omega) \rightarrow \mathbb{R}^{m}, \omega \in(0, \infty]$, of the closed-loop system (18), (22) exists. To this end, we seek to reformulate (18), (22) as an initial value problem of the form

$$
\begin{aligned}
\dot{X}_{I}(t) & =F_{I}\left(t,\left(\begin{array}{c}
X_{I}(t) \\
X_{I I}(t)
\end{array}\right), T_{1}\left(\begin{array}{c}
X_{I} \\
X_{I I}
\end{array}\right)(t)\right), \\
0 & =F_{I I}\left(t,\left(\begin{array}{c}
X_{I}(t) \\
X_{I I}(t)
\end{array}\right), \hat{T}_{2}\left(\begin{array}{c}
X_{I} \\
X_{I I}
\end{array}\right)(t)\right)
\end{aligned}
$$

with

$$
\begin{aligned}
\left.X_{I}\right|_{[-h, 0]} & =\left(y_{1}^{0}, \ldots,\left(\frac{\mathrm{d}}{\mathrm{d} t}\right)^{r_{1}-1} y_{1}^{0}, \ldots,\left(\frac{\mathrm{d}}{\mathrm{d} t}\right)^{r_{q}-1} y_{q}^{0}\right)^{\top}, \\
\left.X_{I I}\right|_{[-h, 0]} & =\left(y_{q+1}^{0}, \ldots, y_{m}^{0}\right)^{\top} .
\end{aligned}
$$

Step 1a: Define, for $i=1, \ldots, q$, and $j=0, \ldots, r_{i}-2$, the sets

$$
\mathscr{D}_{i j}:=\left\{\left(t, e_{i 0}, \ldots, e_{i j}\right) \in \mathbb{R}_{\geq 0} \times \mathbb{R} \times \cdots \times \mathbb{R} \mid\left(t, e_{i \ell}\right) \in \mathscr{F}_{\varphi_{i \ell}}^{1}, \ell=0, \ldots, j\right\},
$$

where $\mathscr{F}_{\varphi_{i \ell}}^{1}$ is as in 20 , and the functions $K_{i j}: \mathscr{D}_{i j} \rightarrow \mathbb{R}$ recursively by

$$
\begin{aligned}
K_{i 0}\left(t, e_{i 0}\right):= & \frac{e_{i 0}}{1-\varphi_{i 0}^{2}(t)\left|e_{i 0}\right|^{2}}, \\
K_{i j}\left(t, e_{i 0}, \ldots, e_{i j}\right):= & \frac{e_{i j}}{1-\varphi_{i j}^{2}(t)\left|e_{i j}\right|^{2}}+\frac{\partial K_{i, j-1}}{\partial t}\left(t, e_{i 0}, \ldots, e_{i, j-1}\right) \\
& +\sum_{\ell=0}^{j-1} \frac{\partial K_{i, j-1}}{\partial e_{\ell j}}\left(t, e_{i 0}, \ldots, e_{i, j-1}\right)\left(e_{i, \ell+1}-\frac{e_{i \ell}}{1-\varphi_{i \ell}^{2}(t)\left|e_{i \ell}\right|^{2}}\right) .
\end{aligned}
$$


Now recall that $\bar{r}=r_{1}+\ldots+r_{q}$ and set

$$
\begin{aligned}
& \mathscr{D}_{I}:=\left\{\left(t, e_{10}, \ldots, e_{1, r_{1}-1}, \ldots, e_{q, r_{q}-1}\right) \in \mathbb{R}_{\geq 0} \times \mathbb{R}^{\bar{r}} \mid\right. \\
&\left.\quad \forall i=1, \ldots, q:\left(t, e_{i 0}, \ldots, e_{i, r_{i}-2}\right) \in \mathscr{D}_{i, r_{i}-2} \wedge\left(t, e_{1, r_{1}-1}, \ldots, e_{q, r_{q}-1}\right) \in \mathscr{F}_{\varphi_{I}}^{q}\right\}, \\
& \mathscr{D}_{I I}:= \mathscr{F}_{\varphi_{I I}}^{m-q}, \\
& \mathscr{D}:=\left\{\left(t, e_{I}, e_{I I}\right) \in \mathbb{R}_{\geq 0} \times \mathbb{R}^{\bar{r}} \times \mathbb{R}^{m-q} \mid\left(t, e_{I}\right) \in \mathscr{D}_{I} \wedge\left(t, e_{I I}\right) \in \mathscr{D}_{I I}\right\} .
\end{aligned}
$$

Choose some interval $I \subseteq \mathbb{R}_{\geq 0}$ with $0 \in I$ and let

$$
\left(e_{10}, \ldots, e_{1, r_{1}-1}, \ldots, e_{q, r_{q}-1}\right): I \rightarrow \mathbb{R}^{\bar{r}}
$$

be sufficiently smooth such that for all $t \in I$ we have

$$
\begin{aligned}
\left(t, e_{10}(t), \ldots, e_{1, r_{1}-1}(t), \ldots, e_{q, r_{q}-1}(t)\right) & \in \mathscr{D}_{I}, \\
\left(t, e_{q+1}(t), \ldots, e_{m}(t)\right) & \in \mathscr{D}_{I I}
\end{aligned}
$$

and $\left(e_{i 0}, \ldots, e_{i, r_{i}-1}\right), i=1, \ldots, q$, satisfies the relations in (22). Then $e_{i}=e_{i 0}$ satisfies, on the interval $I$,

$$
e_{i}^{(j)}=e_{i j}-\sum_{\ell=0}^{j-1}\left(\frac{\mathrm{d}}{\mathrm{d} t}\right)^{j-1-\ell} k_{i \ell} e_{i \ell}, \quad i=1, \ldots, q, j=1, \ldots, r_{i}-1 .
$$

Step $1 b$ : We show by induction that for all $i=1, \ldots, q$, and $j=0, \ldots, r_{i}-2$ we have

$$
\forall t \in I: \sum_{\ell=0}^{j}\left(\frac{\mathrm{d}}{\mathrm{d} t}\right)^{j-\ell}\left(k_{i \ell}(t) e_{i \ell}(t)\right)=K_{i j}\left(t, e_{i 0}(t), \ldots, e_{i j}(t)\right) .
$$

Fix $t \in I$. Equation (32) is obviously true for $j=0$. Assume that $j \in\left\{1, \ldots, r_{i}-2\right\}$ and the statement holds for $j-1$. Then

$$
\begin{aligned}
\sum_{\ell=0}^{j}\left(\frac{\mathrm{d}}{\mathrm{d} t}\right)^{j-\ell}\left(k_{i \ell}(t) e_{i \ell}(t)\right) & =k_{i j}(t) e_{i j}(t)+\frac{\mathrm{d}}{\mathrm{d} t}\left(\sum_{\ell=0}^{j-1}\left(\frac{\mathrm{d}}{\mathrm{d} t}\right)^{j-\ell-1}\left(k_{i \ell}(t) e_{i \ell}(t)\right)\right) \\
& =k_{i j}(t) e_{i j}(t)+\frac{\mathrm{d}}{\mathrm{d} t} K_{i, j-1}\left(t, e_{i 0}(t), \ldots, e_{i, j-1}(t)\right) \\
& =K_{i j}\left(t, e_{i 0}(t), \ldots, e_{i j}(t)\right)
\end{aligned}
$$

Therefore, (32) is shown and, invoking (31), we have for all $i=1, \ldots, q$ and $t \in I$ that

$$
e_{i}^{(j)}(t)=e_{i j}(t)-K_{i, j-1}\left(t, e_{i 0}(t), \ldots, e_{i, j-1}(t)\right), \quad j=1, \ldots, r_{i}-1 .
$$

Step 1c: Define, for $i=1, \ldots, q$,

$$
\tilde{K}_{i 0}: \mathbb{R}_{\geq 0} \times \mathbb{R} \rightarrow \mathbb{R},\left(t, y_{i 0}\right) \mapsto y_{i 0}-y_{\mathrm{ref}, i}(t)
$$

and the set

$$
\tilde{\mathscr{D}}_{i 0}:=\left\{\left(t, y_{i}\right) \in \mathbb{R}_{\geq 0} \times \mathbb{R} \mid\left(t, \tilde{K}_{i 0}\left(t, y_{i}\right)\right) \in \mathscr{D}_{i 0}\right\} .
$$

Furthermore, recursively define the maps

$$
\begin{aligned}
\tilde{K}_{i j}: \tilde{\mathscr{D}}_{i, j-1} \times \mathbb{R} & \rightarrow \mathbb{R}, \\
\left(t, y_{i 0}, \ldots, y_{i j}\right) & \mapsto y_{i j}-y_{\text {ref }, i}^{(j)}(t)+K_{i, j-1}\left(t, \tilde{K}_{i 0}\left(t, y_{i 0}\right), \ldots, \tilde{K}_{i, j-1}\left(t, y_{i 0}, \ldots, y_{i, j-1}\right)\right),
\end{aligned}
$$


for $j=1, \ldots, r_{i}-1$ and the sets

$$
\tilde{\mathscr{D}}_{i j}:=\left\{\left(t, y_{i 0}, \ldots, y_{i j}\right) \in \tilde{\mathscr{D}}_{i, j-1} \times \mathbb{R} \mid\left(t, \tilde{K}_{i 0}\left(t, y_{i 0}\right), \ldots, \tilde{K}_{i j}\left(t, y_{i 0}, \ldots, y_{i j}\right)\right) \in \mathscr{D}_{i j}\right\}
$$

for $j=1, \ldots, r_{i}-2$. Then it follows from (33) and a simple induction that for all $t \in I$, $i=1, \ldots, q$, and $j=0, \ldots, r_{i}-1$ we have

$$
e_{i j}(t)=\tilde{K}_{i j}\left(t, y_{i}(t), \ldots, y_{i}^{(j)}(t)\right) .
$$

Now, define

$$
\begin{aligned}
& \tilde{\mathscr{D}}_{I}:=\left\{\left(t, y_{10}, \ldots, y_{1, r_{1}-1}, \ldots, y_{q, r_{q}-1}\right) \in \mathbb{R}_{\geq 0} \times \mathbb{R}^{\bar{r}}\right. \\
& \forall i=1, \ldots, q:\left(t, y_{i 0}, \ldots, y_{i, r_{i}-1}\right) \in \tilde{\mathscr{D}}_{i, r_{i}-2} \times \mathbb{R} \\
& \left.\wedge\left(t, \tilde{K}_{1, r_{1}-1}\left(t, y_{10}, \ldots, y_{1, r_{1}-1}\right), \ldots, \tilde{K}_{q, r_{q}-1}\left(t, y_{q 0}, \ldots, y_{q, r_{q}-1}\right)\right) \in \mathscr{F}_{\varphi_{I}}^{q}\right\}, \\
& \tilde{\mathscr{D}}_{I I}:=\left\{\left(t, y_{q+1}, \ldots, y_{m}\right) \in \mathbb{R}_{\geq 0} \times \mathbb{R}^{m-q} \mid\left(t, y_{q+1}-y_{\text {ref }, q+1}(t), \ldots, y_{m}-y_{\text {ref }, m}(t)\right) \in \mathscr{D}_{I I}\right\}, \\
& \tilde{K}_{I}: \tilde{\mathscr{D}}_{I} \rightarrow \mathbb{R}^{q},\left(t, y_{10}, \ldots, y_{1, r_{1}-1}, \ldots, y_{q, r_{q}-1}\right) \\
& \mapsto\left(\tilde{K}_{1, r_{1}-1}\left(t, y_{10}, \ldots, y_{1, r_{1}-1}\right), \ldots, \tilde{K}_{q, r_{q}-1}\left(t, y_{q 0}, \ldots, y_{q, r_{q}-1}\right)\right)^{\top},
\end{aligned}
$$

then we find that, for all $t \in I$,

$$
e_{I}(t):=\left(e_{1, r_{1}-1}(t), \ldots, e_{q, r_{q}-1}(t)\right)^{\top}=\tilde{K}_{I}\left(t, y_{1}(t), \ldots, y_{1}^{\left(r_{1}-1\right)}(t), \ldots, y_{q}^{\left(r_{q}-1\right)}(t)\right) .
$$

Further denote, for $t \in I$,

$$
\begin{aligned}
X_{I}(t) & =\left(y_{1}(t), \ldots, y_{1}^{\left(r_{1}-1\right)}(t) \ldots, y_{q}^{\left(r_{q}-1\right)}(t)\right)^{\top}, \quad X_{I I}(t)=\left(y_{q+1}(t), \ldots, y_{m}(t)\right)^{\top}, \\
X_{\mathrm{ref}, I I}(t) & =\left(y_{\mathrm{ref}, q+1}(t), \ldots, y_{\mathrm{ref}, m}(t)\right)^{\top},
\end{aligned}
$$

then

$$
\begin{aligned}
e_{I}(t) & =\tilde{K}_{I}\left(t, X_{I}(t)\right), \\
e_{I I}(t) & :=\left(y_{q+1}(t)-y_{\text {ref }, q+1}(t), \ldots, y_{m}(t)-y_{\text {ref }, m}(t)\right)^{\top}=X_{I I}(t)-X_{\text {ref }, I I}(t)
\end{aligned}
$$

and the feedback $u$ in (22) reads

$$
u(t)=\left(\begin{array}{c}
\frac{-\tilde{K}_{I}\left(t, X_{I}(t)\right)}{1-\varphi_{I}(t)^{2}\left\|\tilde{K}_{I}\left(t, X_{I}(t)\right)\right\|^{2}} \\
\frac{-\hat{k}\left(X_{I I}(t)-X_{\mathrm{ref}, I I}(t)\right)}{1-\varphi_{I I}(t)^{2}\left\|X_{I I}(t)-X_{\mathrm{ref}, I I}(t)\right\|^{2}}
\end{array}\right) .
$$

Step 1d: Now, we set

$$
\begin{aligned}
& H=\operatorname{diag}\left(\left(e_{1}^{\left[r_{1}\right]}\right)^{\top}, \ldots,\left(e_{1}^{\left[r_{q}\right]}\right)^{\top}\right) \in \mathbb{R}^{q \times \bar{r}}, \\
& S=\left[\begin{array}{cc}
H & 0 \\
0 & I_{m-q}
\end{array}\right] \in \mathbb{R}^{m \times(\bar{r}+m-q)},
\end{aligned}
$$

where $e_{1}^{[k]} \in \mathbb{R}^{k}$ is the first canonical unit vector. This construction yields

$$
\forall t \in I: \quad S\left(\begin{array}{c}
X_{I}(t) \\
X_{I I}(t)
\end{array}\right)=y(t)
$$


We define an operator $\hat{T}_{2}: C\left([-h, \infty) \rightarrow \mathbb{R}^{\bar{r}+m-q}\right) \rightarrow C^{1}\left(\mathbb{R}_{\geq 0} \rightarrow \mathbb{R}^{k}\right)$ such that for $\zeta_{1} \in$ $C\left([-h, \infty) \rightarrow \mathbb{R}^{\bar{r}}\right), \zeta_{2} \in C\left([-h, \infty) \rightarrow \mathbb{R}^{m-q}\right)$ we have

$$
\hat{T}_{2}\left(\begin{array}{l}
\zeta_{1} \\
\zeta_{2}
\end{array}\right)(t):=T_{2}\left(S\left(\begin{array}{l}
\zeta_{1} \\
\zeta_{2}
\end{array}\right)\right)(t), \quad t \geq 0 .
$$

Since $T_{2} \in \mathbb{T}_{m, k, h}^{\mathrm{DAE}}$ we obtain that $\hat{T}_{2} \in \mathbb{T}_{\bar{r}+m-q, k, h}^{\mathrm{DAE}}$. Set

$$
\tilde{\mathscr{D}}:=\left\{\left(t, X_{I}, X_{I I}\right) \in \mathbb{R}_{\geq 0} \times \mathbb{R}^{\bar{r}} \times \mathbb{R}^{m-q} \mid\left(t, X_{I}\right) \in \tilde{\mathscr{D}}_{I} \text { and }\left(t, X_{I I}\right) \in \tilde{\mathscr{D}}_{I I}\right\} .
$$

We rewrite $f_{1}$, and $\Gamma_{I}$ from system (18) in vector form

$$
f_{1}=\left(\begin{array}{c}
f_{1}^{1} \\
\vdots \\
f_{1}^{q}
\end{array}\right), \quad \Gamma_{I}=\left(\begin{array}{c}
\Gamma_{I}^{1} \\
\vdots \\
\Gamma_{I}^{q}
\end{array}\right)
$$

with components $f_{1}^{i} \in C\left(\mathbb{R}^{s} \times \mathbb{R}^{k} \rightarrow \mathbb{R}\right)$ and $\Gamma_{I}^{i} \in C\left(\mathbb{R}^{s} \times \mathbb{R}^{k} \rightarrow \mathbb{R}^{1 \times q}\right)$ for $i=1, \ldots, q$. We now define functions $F_{I}: \tilde{\mathscr{D}} \times \mathbb{R}^{k} \rightarrow \mathbb{R}^{\bar{r}}, F_{I I}: \tilde{\mathscr{D}} \times \mathbb{R}^{k} \rightarrow \mathbb{R}^{m-q}$ with

$$
\begin{gathered}
F_{I}: \quad(\underbrace{y_{10}, \ldots, y_{1, r_{1}-1}, \ldots, y_{q, r_{q}-1}}_{=X_{I}}, \underbrace{y_{q+1}, \ldots, y_{m}}_{=X_{I I}}, \eta) \mapsto \\
\left(y_{11}, \ldots, y_{1, r_{1}-1}, f_{1}^{1}\left(d_{1}(t), \eta\right)-\frac{\Gamma_{I}^{1}\left(d_{2}(t), \eta\right) \tilde{K}_{I}\left(t, X_{I}\right)}{1-\varphi_{I}(t)^{2}\left\|\tilde{K}_{I}\left(t, X_{I}\right)\right\|^{2}},\right. \\
\left.\ldots, y_{q, r_{q}-1}, f_{1}^{q}\left(d_{1}(t), \eta\right)-\frac{\Gamma_{I}^{q}\left(d_{2}(t), \eta\right) \tilde{K}_{I}\left(t, X_{I}\right)}{1-\varphi_{I}(t)^{2}\left\|\tilde{K}_{I}\left(t, X_{I}\right)\right\|^{2}}\right),
\end{gathered}
$$

$F_{I I}: \quad(t, \underbrace{y_{10}, \ldots, y_{1, r_{1}-1}, \ldots, y_{q, r_{q}-1}}_{=X_{I}}, \underbrace{y_{q+1}, \ldots, y_{m}}_{=X_{I I}}, \eta) \mapsto$

$$
\begin{aligned}
\left(f_{2}\left(X_{I}, X_{I I}\right)+f_{3}\left(d_{3}(t)\right.\right. & , \eta)-\frac{\Gamma_{I I}\left(d_{4}(t), \eta\right) \tilde{K}_{I}\left(t, X_{I}\right)}{1-\varphi_{I}(t)^{2}\left\|\tilde{K}_{I}\left(t, X_{I}\right)\right\|^{2}} \\
& \left.-f_{4}\left(d_{5}(t), \eta\right) \frac{\hat{k}\left(X_{I I}-X_{\mathrm{ref}, I I}(t)\right)}{1-\varphi_{I I}(t)^{2}\left\|X_{I I}-X_{\mathrm{ref}, I I}(t)\right\|^{2}}\right) .
\end{aligned}
$$

Then the closed-loop system (18), 22) is equivalent to (29).

Step 1e: In order to show that 29] has a solution we take the derivative of the second equation and rewrite it appropriately. First observe that since $T_{2} \in \mathbb{T}_{m, k, h}^{\mathrm{DAE}}$ there exist $z \in$ $C\left(\mathbb{R}^{m} \times \mathbb{R}^{k} \rightarrow \mathbb{R}^{k}\right)$ and $\tilde{T}_{2} \in \mathbb{T}_{m, k, h}$ such that

$$
\forall \zeta \in C\left([-h, \infty) \rightarrow \mathbb{R}^{m}\right) \forall t \geq 0: \frac{\mathrm{d}}{\mathrm{d} t}\left(T_{2} \zeta\right)(t)=z\left(\zeta(t),\left(\tilde{T}_{2} \zeta\right)(t)\right) .
$$

Now define the operator $\hat{T}_{3}: C\left([-h, \infty) \rightarrow \mathbb{R}^{\bar{r}+m-q}\right) \rightarrow C\left(\mathbb{R}_{\geq 0} \rightarrow \mathbb{R}^{k}\right)$ by the property that for $\zeta_{1} \in C\left([-h, \infty) \rightarrow \mathbb{R}^{\bar{r}}\right), \zeta_{2} \in C\left([-h, \infty) \rightarrow \mathbb{R}^{m-q}\right)$ we have

$$
\hat{T}_{3}\left(\begin{array}{l}
\zeta_{1} \\
\zeta_{2}
\end{array}\right)(t):=\tilde{T}_{2}\left(S\left(\begin{array}{l}
\zeta_{1} \\
\zeta_{2}
\end{array}\right)\right)(t), \quad t \geq 0,
$$


then $\hat{T}_{3} \in \mathbb{T}_{\bar{r}+m-q, k, h}$. A differentiation of the second equation in 29] yields

$$
\begin{aligned}
0= & \frac{\partial F_{I I}}{\partial t}\left(t,\left(\begin{array}{c}
X_{I}(t) \\
X_{I I}(t)
\end{array}\right), \hat{T}_{2}\left(\begin{array}{c}
X_{I} \\
X_{I I}
\end{array}\right)(t)\right)+\frac{\partial F_{I I}}{\partial X_{I}}\left(t,\left(\begin{array}{c}
X_{I}(t) \\
X_{I I}(t)
\end{array}\right), \hat{T}_{2}\left(\begin{array}{c}
X_{I} \\
X_{I I}
\end{array}\right)(t)\right) \dot{X}_{I}(t) \\
& +\frac{\partial F_{I I}}{\partial X_{I I}}\left(t,\left(\begin{array}{c}
X_{I}(t) \\
X_{I I}(t)
\end{array}\right), \hat{T}_{2}\left(\begin{array}{c}
X_{I} \\
X_{I I}
\end{array}\right)(t)\right) \dot{X}_{I I}(t) \\
& +\frac{\partial F_{I I}}{\partial \eta}\left(t,\left(\begin{array}{c}
X_{I}(t) \\
X_{I I}(t)
\end{array}\right), \hat{T}_{2}\left(\begin{array}{c}
X_{I} \\
X_{I I}
\end{array}\right)(t)\right) \frac{\mathrm{d}}{\mathrm{d} t}\left(\hat{T}_{2}\left(\begin{array}{c}
X_{I} \\
X_{I I}
\end{array}\right)\right)(t),
\end{aligned}
$$

by which, using the first equation in 29) and

$$
\frac{\mathrm{d}}{\mathrm{d} t}\left(\hat{T}_{2}\left(\begin{array}{c}
X_{I} \\
X_{I I}
\end{array}\right)\right)(t)=z\left(S\left(\begin{array}{c}
X_{I}(t) \\
X_{I I}(t)
\end{array}\right), \hat{T}_{3}\left(\begin{array}{c}
X_{I} \\
X_{I I}
\end{array}\right)(t)\right),
$$

we obtain

$$
\begin{aligned}
\frac{\partial F_{I I}}{\partial X_{I I}}\left(t,\left(\begin{array}{c}
X_{I}(t) \\
X_{I I}(t)
\end{array}\right), \hat{T}_{2}\left(\begin{array}{c}
X_{I} \\
X_{I I}
\end{array}\right)(t)\right) \dot{X}_{I I}(t) \\
\quad=\hat{F}_{I I}\left(t,\left(\begin{array}{c}
X_{I}(t) \\
X_{I I}(t)
\end{array}\right), T_{1}\left(\begin{array}{c}
X_{I} \\
X_{I I}
\end{array}\right)(t), \hat{T}_{2}\left(\begin{array}{c}
X_{I} \\
X_{I I}
\end{array}\right)(t), \hat{T}_{3}\left(\begin{array}{c}
X_{I} \\
X_{I I}
\end{array}\right)(t)\right)
\end{aligned}
$$

for some $\hat{F}_{I I}: \tilde{\mathscr{D}} \times \mathbb{R}^{3 k} \rightarrow \mathbb{R}^{m-q}$. We show that the matrix

$$
\begin{aligned}
\frac{\partial F_{I I}}{\partial X_{I I}}\left(t, X_{I}, X_{I I}, \eta\right)=\frac{\partial f_{2}\left(X_{I}, X_{I I}\right)}{\partial X_{I I}}-\frac{\hat{k} f_{4}\left(d_{5}(t), \eta\right)}{1-\varphi_{I I}(t)^{2}\left\|X_{I I}-X_{\mathrm{ref}, I I}(t)\right\|^{2}} \cdot \\
\cdot\left(I_{m-q}+\frac{2 \varphi_{I I}(t)^{2}\left(X_{I I}-X_{\mathrm{ref}, I I}(t)\right)\left(X_{I I}-X_{\mathrm{ref}, I I}(t)\right)^{\top}}{1-\varphi_{I I}(t)^{2}\left\|X_{I I}-X_{\mathrm{ref}, I I}(t)\right\|^{2}}\right)
\end{aligned}
$$

is invertible for all $\left(t, X_{I}, X_{I I}, \eta\right) \in \tilde{\mathscr{D}} \times \mathbb{R}^{k}$ : The symmetry and positive semi-definiteness of

$$
\mathscr{G}\left(t, X_{I I}\right):=\frac{2 \varphi_{I I}(t)^{2}\left(X_{I I}-X_{\mathrm{ref}, I I}(t)\right)\left(X_{I I}-X_{\mathrm{ref}, I I}(t)\right)^{\top}}{1-\varphi_{I I}(t)^{2}\left\|X_{I I}-X_{\mathrm{ref}, I I}(t)\right\|^{2}}
$$

implies positive definiteness (and hence invertibility) of $I_{m-q}+\mathscr{G}\left(t, X_{I I}\right)$ for all $\left(t, X_{I I}\right) \in \tilde{\mathscr{D}}_{I I}$, and by [5, Lem. 3.3] we further have

$$
\left\|\left(I_{m-q}+\mathscr{G}\left(t, X_{I I}\right)\right)^{-1}\right\| \leq 1
$$

Therefore, according to (24) and Assumption 3.2 (iv), we have for all $\left(t, X_{I}, X_{I I}, \eta\right) \in \tilde{\mathscr{D}} \times \mathbb{R}^{k}$ that

$$
\begin{array}{r}
\left\|\left(1-\varphi_{I I}(t)^{2}\left\|X_{I I}-X_{\mathrm{ref}, I I}(t)\right\|^{2}\right) \hat{k}^{-1}\left[f_{4}\left(d_{5}(t), \eta\right)\right]^{-1}\left(I_{m-q}+\mathscr{G}\left(t, X_{I I}\right)\right)^{-1} \frac{\partial f_{2}\left(X_{I}, X_{I I}\right)}{\partial X_{I I}}\right\| \\
\leq \hat{k}^{-1} \alpha^{-1}\left\|\frac{\partial f_{2}\left(X_{I}, X_{I I}\right)}{\partial X_{I I}}\right\| \stackrel{[24]}{<} 1 .
\end{array}
$$

This implies invertibility of $\frac{\partial F_{I I}}{\partial X_{I I}}\left(t, X_{I}, X_{I I}, \eta\right)$ for all $\left(t, X_{I}, X_{I I}, \eta\right) \in \tilde{\mathscr{D}} \times \mathbb{R}^{k}$. With $\tilde{F}_{I I}: \tilde{\mathscr{D}} \times$ $\mathbb{R}^{3 k} \rightarrow \mathbb{R}^{m-q}$ defined by

$$
\tilde{F}_{I I}\left(t, X_{I}, X_{I I}, \eta_{1}, \eta_{2}, \eta_{3}\right):=\left(\frac{\partial F_{I I}}{\partial X_{I I}}\left(t, X_{I}, X_{I I}, \eta_{2}\right)\right)^{-1} \hat{F}_{I I}\left(t, X_{I}, X_{I I}, \eta_{1}, \eta_{2}, \eta_{3}\right)
$$


and the first equation in 29] we obtain the ODE

$$
\begin{aligned}
& \dot{X}_{I}(t)=F_{I}\left(t,\left(\begin{array}{c}
X_{I}(t) \\
X_{I I}(t)
\end{array}\right), T_{1}\left(\begin{array}{c}
X_{I} \\
X_{I I}
\end{array}\right)(t)\right) \\
& \dot{X}_{I I}(t)=\tilde{F}_{I I}\left(t,\left(\begin{array}{c}
X_{I}(t) \\
X_{I I}(t)
\end{array}\right), T_{1}\left(\begin{array}{c}
X_{I} \\
X_{I I}
\end{array}\right)(t), \hat{T}_{2}\left(\begin{array}{c}
X_{I} \\
X_{I I}
\end{array}\right)(t), \hat{T}_{3}\left(\begin{array}{c}
X_{I} \\
X_{I I}
\end{array}\right)(t)\right),
\end{aligned}
$$

with initial conditions 30 .

Step 1f: Consider the initial value problem (36), 30), then we have $\left(0, X_{I}(0), X_{I I}(0)\right) \in \tilde{\mathscr{D}}, F_{I}$ is measurable in $t$, continuous in $\left(X_{I}, X_{I I}, \eta\right)$, and locally essentially bounded, and $\tilde{F}_{I I}$ is measurable in $t$, continuous in $\left(X_{I}, X_{I I}, \eta_{1}, \eta_{2}, \eta_{3}\right)$, and locally essentially bounded. Therefore, by [21, Theorem B.11 we obtain existence of solutions to (36), and every solution can be extended to a maximal solution. Furthermore, for a maximal solution $\left(X_{I}, X_{I I}\right):[-h, \omega) \rightarrow$ $\mathbb{R}^{\bar{r}+m-q}, \omega \in(0, \infty]$, of (36), (30) the closure of the graph of this solution is not a compact subset of $\tilde{\mathscr{D}}$.

We show that $\left(X_{I}, X_{I I}\right)$ is also a maximal solution of $(29)$. Since $\left(X_{I}, X_{I I}\right)$ is particular satisfies, by construction,

$$
\forall t \in[0, \omega): 0=\frac{\mathrm{d}}{\mathrm{d} t} F_{I I}\left(t,\left(\begin{array}{c}
X_{I}(t) \\
X_{I I}(t)
\end{array}\right), \hat{T}_{2}\left(\begin{array}{c}
X_{I} \\
X_{I I}
\end{array}\right)(t)\right),
$$

there exists $c \in \mathbb{R}^{m-q}$ such that

$$
\forall t \in[0, \omega): c=F_{I I}\left(t,\left(\begin{array}{c}
X_{I}(t) \\
X_{I I}(t)
\end{array}\right), \hat{T}_{2}\left(\begin{array}{c}
X_{I} \\
X_{I I}
\end{array}\right)(t)\right) .
$$

Invoking (30), the definition of $F_{I I}$ and $\hat{T}_{2}$, and the consistency condition (26) we may infer that $c=0$. Therefore, $\left(X_{I}, X_{I I}\right)$ is a solution of [29]. Furthermore, $\left(X_{I}, X_{I I}\right)$ is also a maximal solution of (29), since any right extension would be a solution of (36) following the procedure in Step 1e, a contradiction.

define

Recall $X_{I}(t)=\left(y_{1}(t), \ldots, y_{1}^{\left(r_{1}-1\right)}(t) \ldots, y_{q}^{\left(r_{q}-1\right)}(t)\right)^{\top}, X_{I I}(t)=\left(y_{q+1}(t), \ldots, y_{m}(t)\right)^{\top}$ and

$$
\left(e_{10}, \ldots, e_{1, r_{1}-1}, \ldots, e_{q, r_{q}-1}, e_{q+1}, \ldots, e_{m}\right):[0, \omega) \rightarrow \mathbb{R}^{\bar{r}+m-q}
$$

by

$$
\begin{aligned}
e_{i j}(t) & =\tilde{K}_{i j}\left(t, y_{i}(t), \ldots, y_{i}^{(j)}(t)\right), & & \text { for } i=1, \ldots, q \text { and } j=0, \ldots, r_{i}-1, \\
e_{i}(t) & =y_{i}(t)-y_{\mathrm{ref}, i}(t), & & \text { for } i=q+1, \ldots, m,
\end{aligned}
$$

then the closure of the graph of the function in 37 is not a compact subset of $\mathscr{D}$.

Step 2: We show boundedness of the gain functions $k_{I}(\cdot), k_{I I}(\cdot)$ and $k_{i j}(\cdot)$ as in 22) on $[0, \omega)$. This also proves (28).

Step 2a: The proof of boundedness of $k_{i j}(\cdot)$ for $i=1, \ldots, q, j=0, \ldots, r_{i}-2$ on $[0, \omega)$ is analogous to Step 2a of the proof of [7] Thm. 3.1] and hence omitted.

Step $2 b$ : We prove by induction that there exist constants $M_{i j}^{\ell}, N_{i j}^{\ell}, K_{i j}^{\ell}>0$ such that, for all $t \in[0, \omega)$,

$$
\left|\left(\frac{\mathrm{d}}{\mathrm{d} t}\right)^{\ell}\left[k_{i j}(t) e_{i j}(t)\right]\right| \leq M_{i j}^{\ell}, \quad\left|\left(\frac{\mathrm{d}}{\mathrm{d} t}\right)^{\ell} e_{i j}(t)\right| \leq N_{i j}^{\ell}, \quad\left|\left(\frac{\mathrm{d}}{\mathrm{d} t}\right)^{\ell} k_{i j}(t)\right| \leq K_{i j}^{\ell},
$$

\footnotetext{
1 In [21] a domain $\mathscr{D} \subseteq \mathbb{R}_{\geq 0} \times \mathbb{R}$ is considered, but the generalization to the higher dimensional case is straightforward.
} 
for $i=1, \ldots, q, j=0, \ldots, r_{i}-2$, and $\ell=0, \ldots, r_{i}-1-j$.

First, we may infer from Step $2 a$ that $k_{i j}(\cdot)$, for $i=1, \ldots, q, j=0, \ldots, r_{i}-2$, are bounded. Furthermore, $e_{i j}$ are bounded since they evolve in the respective performance funnels. Therefore, for each $i=1, \ldots, q$ and $j=0, \ldots, r_{i}-2$, (38) is true whenever $\ell=0$. Fix $i \in\{1, \ldots, q\}$. We prove (38) for $j=r_{i}-2$ and $\ell=1$ :

$$
\begin{aligned}
\dot{e}_{i, r_{i}-2}(t)= & e_{i, r_{i}-1}(t)-k_{i, r_{i}-2}(t) e_{i, r_{i}-2}(t), \\
\dot{k}_{i, r_{i}-2}(t)= & 2 k_{i, r_{i}-2}^{2}(t)\left(\varphi_{i, r_{i}-2}^{2}(t) e_{i, r_{i}-2}(t) \dot{e}_{i, r_{i}-2}(t)\right. \\
& \left.+\varphi_{i, r_{i}-2}(t) \dot{\varphi}_{i, r_{i}-2}(t)\left|e_{i, r_{i}-2}(t)\right|^{2}\right), \\
\frac{\mathrm{d}}{\mathrm{d} t}\left[k_{i, r_{i}-2}(t) e_{i, r_{i}-2}(t)\right]= & \dot{k}_{i, r_{i}-2}(t) e_{i, r_{i}-2}(t)+k_{i, r_{i}-2}(t) \dot{e}_{i, r_{i}-2}(t) .
\end{aligned}
$$

Boundedness of $k_{i, r_{i}-2}, \varphi_{i, r_{i}-2}, \dot{\varphi}_{i, r_{i}-2}, e_{i, r_{i}-2}$ together with the above equations implies that $\dot{e}_{i, r_{i}-2}(t), \dot{k}_{i, r_{i}-2}(t)$ and $\frac{\mathrm{d}}{\mathrm{d} t}\left[k_{i, r_{i}-2}(t) e_{i, r_{i}-2}(t)\right]$ are bounded. Now consider indices $s \in$ $\left\{0, \ldots, r_{i}-3\right\}$ and $l \in\left\{0, \ldots, r_{i}-1-s\right\}$ and assume that $(38)$ is true for all $j=s+1, \ldots, r_{i}-$ 2 and all $\ell=0, \ldots, r_{i}-1-j$ as well as for $j=s$ and all $\ell=0, \ldots, l-1$. We show that it is true for $j=s$ and $\ell=l$ :

$$
\begin{aligned}
\left(\frac{\mathrm{d}}{\mathrm{d} t}\right)^{l} e_{i s}(t) & =\left(\frac{\mathrm{d}}{\mathrm{d} t}\right)^{l-1}\left[e_{i, s+1}(t)-k_{i s}(t) e_{i s}(t)\right] \\
& =\left(\frac{\mathrm{d}}{\mathrm{d} t}\right)^{l-1} e_{i, s+1}(t)-\left(\frac{\mathrm{d}}{\mathrm{d} t}\right)^{l-1}\left[k_{i s}(t) e_{i s}(t)\right] \\
\left(\frac{\mathrm{d}}{\mathrm{d} t}\right)^{l} k_{i s}(t) & =\left(\frac{\mathrm{d}}{\mathrm{d} t}\right)^{l-1}\left(2 k_{i s}^{2}(t)\left(\varphi_{i s}^{2}(t) e_{i s}(t) \dot{e}_{i s}(t)+\varphi_{i s}(t) \dot{\varphi}_{i s}(t)\left|e_{i s}(t)\right|^{2}\right)\right), \\
\left(\frac{\mathrm{d}}{\mathrm{d} t}\right)^{l}\left[k_{i s}(t) e_{i s}(t)\right] & =\left(\frac{\mathrm{d}}{\mathrm{d} t}\right)^{l-1}\left(\dot{k}_{i s}(t) e_{i s}(t)+k_{i s}(t) \dot{e}_{i s}(t)\right) .
\end{aligned}
$$

Then, successive application of the product rule and using the induction hypothesis as wells as the fact that $\varphi_{i s}, \dot{\varphi}_{i s}, \ldots, \varphi_{i s}^{\left(r_{i}-s\right)}$ are bounded, yields that the above terms are bounded. Therefore, the proof of 38 is complete.

It follows from (38) and (31) that, for all $i=1, \ldots, q$ and $j=0, \ldots, r_{i}-1, e_{i}^{(j)}$ is bounded on $[0, \omega)$.

Step 2c: We show that $k_{I}(\cdot)$ as in (22) is bounded. It follows from (31) that, for $i=1, \ldots, q$,

$$
e_{i}^{\left(r_{i}\right)}(t)=\dot{e}_{i, r_{i}-1}(t)-\sum_{j=0}^{r_{i}-2}\left(\frac{\mathrm{d}}{\mathrm{d} t}\right)^{r_{i}-1-j}\left[k_{i j}(t) e_{i j}(t)\right] .
$$

Then we find that by 29 )

$$
\begin{aligned}
\dot{e}_{I}(t)= & f_{1}\left(d_{1}(t), T_{1}\left(y_{1}, \ldots, y_{1}^{\left(r_{1}-1\right)}, \ldots, y_{q}^{\left(r_{q}-1\right)}, y_{q+1}, \ldots, y_{m}\right)(t)\right) \\
& -\Gamma_{I}\left(d_{1}(t), T_{1}\left(y_{1}, \ldots, y_{1}^{\left(r_{1}-1\right)}, \ldots, y_{q}^{\left(r_{q}-1\right)}, y_{q+1}, \ldots, y_{m}\right)(t)\right) k_{I}(t) e_{I}(t) \\
& +\left(\begin{array}{c}
\sum_{j=0}^{r_{1}-2}\left(\frac{\mathrm{d}}{\mathrm{d} t}\right)^{r_{1}-1-j} k_{1 j}(t) e_{1 j}(t) \\
\sum_{j=0}^{r_{2}-2}\left(\frac{\mathrm{d}}{\mathrm{d} t}\right)^{r_{2}-1-j} k_{2 j}(t) e_{2 j}(t) \\
\vdots \\
\sum_{j=0}^{r_{q}-2}\left(\frac{\mathrm{d}}{\mathrm{d} t}\right)^{r_{q}-1-j} k_{q j}(t) e_{q j}(t)
\end{array}\right)-\left(\begin{array}{c}
y_{\mathrm{ref}, 1}^{\left(r_{1}\right)}(t) \\
\vdots \\
y_{\mathrm{ref}, q}^{\left(r_{q}\right)}(t)
\end{array}\right) .
\end{aligned}
$$


Again we use $X_{I}(t)=\left(y_{1}(t), \ldots, y_{1}^{\left(r_{1}-1\right)}(t) \ldots, y_{q}^{\left(r_{q}-1\right)}(t)\right)^{\top}, X_{I I}(t)=\left(y_{q+1}(t), \ldots, y_{m}(t)\right)^{\top}$ and we set, for $t \in[0, \omega)$,

$$
\begin{aligned}
\hat{F}_{I}(t):= & f_{1}\left(d_{1}(t), T_{1}\left(X_{I}, X_{I I}\right)(t)\right) \\
& +\left(\begin{array}{c}
\sum_{j=0}^{r_{1}-2}\left(\frac{\mathrm{d}}{\mathrm{d} t}\right)^{r_{1}-1-j} k_{1 j}(t) e_{1 j}(t) \\
\sum_{j=0}^{r_{2}-2}\left(\frac{\mathrm{d}}{\mathrm{d} t}\right)^{r_{2}-1-j} k_{2 j}(t) e_{2 j}(t) \\
\vdots \\
\sum_{j=0}^{r_{q}-2}\left(\frac{\mathrm{d}}{\mathrm{d} t}\right)^{r_{q}-1-j} k_{q j}(t) e_{q j}(t)
\end{array}\right)-\left(\begin{array}{c}
y_{\mathrm{ref}, 1}^{\left(r_{1}\right)}(t) \\
\vdots \\
y_{\mathrm{ref}, q}^{\left(r_{q}\right)}(t)
\end{array}\right) .
\end{aligned}
$$

We obtain from (38) and (31) that $e_{i}^{(j)}$ is bounded on the interval $[0, \omega)$ for $i=1, \ldots, q$ and $j=0, \ldots, r_{i}-2$. Furthermore, $e_{I}$ evolves in the performance funnel $\mathscr{F}_{\varphi_{I}}^{q}$, thus $\left|e_{i, r_{i}-1}(t)\right|^{2} \leq$ $\left\|e_{I}(t)\right\|^{2}<\varphi_{I}(t)^{-1}$ for all $t \in[0, \omega)$, so $e_{i, r_{i}-1}$ is bounded on $[0, \omega)$ for $i=1, \ldots, q$. Invoking boundedness of $y_{\text {ref }, i}^{(j)}$ yields boundedness of $y_{i}^{(j)}$ for $i=1, \ldots, q, j=0, \ldots, r_{i}-1$. Then the bounded-input, bounded-output property of $T_{1}$ in Definition 3.1 (iii) implies that $T_{1}\left(X_{I}, X_{I I}\right)$ is bounded by

$$
M_{T_{1}}:=\left\|\left.T_{1}\left(X_{I}, X_{I I}\right)\right|_{[0, \omega)}\right\|_{\infty} .
$$

This property together with $(38)$, continuity of $f_{1}$ and boundedness of $d_{1}$ yields that $\hat{F}_{I}(\cdot)$ is bounded on $[0, \omega)$. In other words, there exists some $M_{\hat{F}_{I}}>0$ such that $\left\|\left.\hat{F}_{I}\right|_{[0, \omega)}\right\|_{\infty} \leq M_{\hat{F}_{I}}$. Now define the compact set

$$
\Omega=\left\{\left(\delta, \eta, e_{I}\right) \in \mathbb{R}^{s} \times \mathbb{R}^{k} \times \mathbb{R}^{q}\left|\|\delta\| \leq\left\|\left.d_{2}\right|_{[0, \omega)}\right\|_{\infty},\|\eta\| \leq M_{T_{1}},\left\|e_{I}\right\|=1\right\},\right.
$$

then, since $\Gamma_{I}+\Gamma_{I}^{\top}$ is pointwise positive definite by Assumption 3.2 (i) and the map

$$
\Omega \ni\left(\delta, \eta, e_{I}\right) \mapsto e_{I}^{\top}\left(\Gamma_{I}(\delta, \eta)+\Gamma_{I}(\delta, \eta)^{\top}\right) e_{I} \in \mathbb{R}_{>0}
$$

is continuous, it follows that there exists $\gamma>0$ such that

$$
\forall\left(\delta, \eta, e_{I}\right) \in \Omega: \quad e_{I}^{\top}\left(\Gamma_{I}(\delta, \eta)+\Gamma_{I}(\delta, \eta)^{\top}\right) e_{I} \geq \gamma .
$$

Therefore, we have for all $t \in[0, \omega)$ that

$$
e_{I}(t)^{\top}\left(\Gamma_{I}\left(d_{1}(t), T_{1}\left(X_{I}, X_{I I}\right)(t)\right)+\Gamma_{I}\left(d_{1}(t), T_{1}\left(X_{I}, X_{I I}\right)(t)\right)^{\top}\right) e_{I}(t) \geq \gamma\left\|e_{I}(t)\right\|^{2} .
$$

Now, set $\psi_{I}(t):=\varphi_{I}(t)^{-1}$ for $t \in(0, \omega)$, let $T_{I} \in(0, \omega)$ be arbitrary but fixed and set $\lambda_{I}:=\inf _{t \in(0, \omega)} \psi_{I}(t)$. Since $\dot{\varphi}_{I}$ is bounded and $\liminf _{t \rightarrow \infty} \varphi_{I}(t)>0$ we find that $\left.\frac{\mathrm{d}}{\mathrm{d} t} \psi_{I}\right|_{[0, \omega)}$ is bounded and hence $\left.\psi_{I}\right|_{[0, \omega)}$ is Lipschitz continuous with Lipschitz bound $L_{I}>0$. Choose $\varepsilon_{I}>0$ small enough such that

$$
\begin{aligned}
\varepsilon_{I} & \leq \min \left\{\frac{\lambda_{I}}{2}, \inf _{t \in\left(0, T_{I}\right]}\left(\psi_{I}(t)-\left\|e_{I}(t)\right\|\right)\right\} \\
\text { and } \quad L_{I} & \leq \frac{\lambda_{I}^{2}}{8 \varepsilon_{I}} \gamma-M_{\hat{F}_{I}},
\end{aligned}
$$


We show that

$$
\forall t \in(0, \omega): \psi_{I}(t)-\left\|e_{I}(t)\right\| \geq \varepsilon_{I} .
$$

By definition of $\varepsilon_{I}$ this holds on $\left(0, T_{I}\right]$. Seeking a contradiction suppose that

$$
\exists t_{I, 1} \in\left[T_{I}, \omega\right): \psi_{I}\left(t_{I, 1}\right)-\left\|e_{I}\left(t_{I, 1}\right)\right\|<\varepsilon_{I} .
$$

Set $t_{I, 0}=\max \left\{t \in\left[T_{I}, t_{I, 1}\right) \mid \psi_{I}(t)-\left\|e_{I}(t)\right\|=\varepsilon_{I}\right\}$. Then, for all $t \in\left[t_{I, 0}, t_{I, 1}\right]$, we have

$$
\begin{aligned}
\psi_{I}(t)-\left\|e_{I}(t)\right\| & \leq \varepsilon_{I}, \\
\left\|e_{I}(t)\right\| \geq \psi_{I}(t)-\varepsilon_{I} & \geq \frac{\lambda_{I}}{2}, \\
k_{I}(t)=\frac{1}{1-\varphi_{I}^{2}(t)\left\|e_{I}(t)\right\|^{2}} & \geq \frac{\lambda_{I}}{2 \varepsilon_{I}} .
\end{aligned}
$$

Then it follows from (39) and (40) that for all $t \in\left[t_{I, 0}, t_{I, 1}\right]$,

$$
\begin{aligned}
& \frac{1}{2} \frac{\mathrm{d}}{\mathrm{d} t}\left\|e_{I}(t)\right\|^{2}=\frac{1}{2}\left(e_{I}^{\top}(t) \dot{e}_{I}(t)+\dot{e}_{I}^{\top}(t) e_{I}(t)\right) \\
& =e_{I}^{\top}(t)\left(\hat{F}_{I}(t)-\frac{1}{2}\left(\Gamma_{I}\left(d_{1}(t), T_{1}\left(X_{I}, X_{I I}\right)(t)\right)+\Gamma_{I}\left(d_{1}(t), T_{1}\left(X_{I}, X_{I I}\right)(t)\right)^{\top}\right) k_{I}(t) e_{I}(t)\right) \\
& \leq\left(M_{\hat{F}_{I}}-\frac{\lambda_{I}^{2}}{8 \varepsilon_{I}} \gamma\right)\left\|e_{I}(t)\right\| \stackrel{41}{\leq}-L_{I}\left\|e_{I}(t)\right\| .
\end{aligned}
$$

Then, using $\left\|e_{I}(t)\right\| \geq \frac{\lambda_{I}}{2}>0$ for all $t \in\left[t_{I, 0}, t_{I, 1}\right]$,

$$
\begin{aligned}
\left\|e_{I}\left(t_{I, 1}\right)\right\|-\left\|e_{I}\left(t_{I, 0}\right)\right\| & =\int_{t_{I, 0}}^{t_{I, 1}} \frac{1}{2}\left\|e_{I}(t)\right\|^{-1} \frac{\mathrm{d}}{\mathrm{d} t}\left\|e_{I}(t)\right\|^{2} \mathrm{~d} t \\
& \leq-L_{I}\left(t_{I, 1}-t_{I, 0}\right) \leq-\left|\psi_{I}\left(t_{I, 1}\right)-\psi_{I}\left(t_{I, 0}\right)\right| \leq \psi_{I}\left(t_{I, 1}\right)-\psi_{I}\left(t_{I, 0}\right),
\end{aligned}
$$

and thus we obtain $\varepsilon_{I}=\psi_{I}\left(t_{I, 0}\right)-\left\|e_{I}\left(t_{I, 0}\right)\right\| \leq \psi_{I}\left(t_{I, 1}\right)-\left\|e_{I}\left(t_{I, 1}\right)\right\|<\varepsilon_{I}$, a contradiction. Step $2 d$ : We show that $k_{I I}(\cdot)$ as in (22) is bounded. Seeking a contradiction, we assume that $k_{I I}(t) \rightarrow \infty$ for $t \rightarrow \omega$. Set, for $t \in[0, \omega)$,

$$
\check{F}_{I I}(t):=f_{2}\left(X_{I}(t), X_{I I}(t)\right)+f_{3}\left(d_{3}(t),\left(T_{2} y\right)(t)\right)-\Gamma_{I I}\left(d_{4}(t),\left(T_{2} y\right)(t)\right) k_{I}(t) e_{I}(t) .
$$

Since $k_{I}$ is bounded on $[0 \omega)$ by Step $2 \mathrm{c}$, it follows from Step $2 \mathrm{~b}$, boundedness of $T_{2}(y), d_{3}$ and $d_{4}$ and continuity of $f_{2}, f_{3}$ and $\Gamma_{I I}$ that $\check{F}_{I I}(\cdot)$ is bounded on $[0, \omega)$. By $(29)$ we have

$$
0=\breve{F}_{I I}(t)-f_{4}\left(d_{5}(t),\left(T_{2} y\right)(t)\right) k_{I I}(t) e_{I I}(t) .
$$

We show that $e_{I I}(t) \rightarrow 0$ for $t \rightarrow \omega$. Seeking a contradiction, assume that there exist $\kappa>0$ and a sequence $\left(t_{n}\right) \subset \mathbb{R}_{\geq 0}$ with $t_{n} \nearrow \omega$ such that $\left\|e_{I I}\left(t_{n}\right)\right\| \geq \kappa$ for all $n \in \mathbb{N}$. Then, from (44) we obtain, for all $t \in[0, \omega)$,

$$
\left\|\check{F}_{I I}(t)\right\|=\left\|f_{4}\left(d_{5}(t),\left(T_{2} y\right)(t)\right) k_{I I}(t) e_{I I}(t)\right\|=\left|f_{4}\left(d_{5}(t),\left(T_{2} y\right)(t)\right)\right| \cdot\left|k_{I I}(t)\right| \cdot\left\|e_{I I}(t)\right\| .
$$

Since $k_{I I}(t) \rightarrow \infty$ for $t \rightarrow \omega,\left\|e_{I I}\left(t_{n}\right)\right\| \geq \kappa$ and $f_{4}\left(d_{5}\left(t_{n}\right),\left(T_{2} y\right)\left(t_{n}\right)\right) \geq \alpha$, we find that

$$
\left\|\check{F}_{I I}\left(t_{n}\right)\right\| \geq \alpha \kappa k_{I I}\left(t_{n}\right) \rightarrow \infty \text { for } n \rightarrow \infty,
$$


which contradicts boundedness of $\check{F}_{I I}(\cdot)$.

Hence, we have $e_{I I}(t) \rightarrow 0$ for $t \rightarrow \omega$, by which $\lim _{t \rightarrow \infty} \varphi_{I I}(t)^{2}\left\|e_{I I}(t)\right\|^{2}=0$ because $\varphi_{I I}(\cdot)$ is bounded. This leads to the contradiction $\lim _{t \rightarrow \infty} k_{I I}(t)=\hat{k}$, thus $k_{I I}(\cdot)$ is bounded. Step 3: We show that $\omega=\infty$. Seeking a contradiction, we assume that $\omega<\infty$. Then, since $e_{I}, e_{I I}, k_{I}, k_{I I}$ and $e_{i j}, k_{i j}$ are bounded for $i=1, \ldots, q, j=0, \ldots, r_{i}-2$ by Step 2, it follows that the closure of the graph of the function in 37 is a compact subset of $\mathscr{D}$, which is a contradiction. This finishes the proof of the theorem.

\section{Simulations}

In this section we illustrate the application of the funnel controller (22) by considering the following academic example:

$$
\begin{aligned}
\ddot{y}_{1}(t)=- & \sin y_{1}(t)+y_{1}(t) \dot{y}_{1}(t)+y_{2}(t)^{2} \\
& +\dot{y}_{1}(t)^{2} T\left(y_{1}, y_{2}\right)(t)+\left(y_{1}(t)^{2}+y_{2}(t)^{4}+1\right) u_{I}(t), \\
0=y_{1}(t)^{3}+y_{1}(t) \dot{y}_{1}(t)^{3}+y_{2}(t)+T\left(y_{1}, y_{2}\right)(t)+ & \\
& +T\left(y_{1}, y_{2}\right)(t) u_{I}(t)+u_{I I}(t),
\end{aligned}
$$

where $T: C\left(\mathbb{R}_{\geq 0} \rightarrow \mathbb{R}^{m}\right) \rightarrow C^{1}\left(\mathbb{R}_{\geq 0} \rightarrow \mathbb{R}\right)$ is given by

$$
T\left(y_{1}, y_{2}\right)(t):=e^{-2 t} \eta^{0}+\int_{0}^{t} e^{-2(t-s)}\left(2 y_{1}(s)-y_{2}(s)\right) d s, \quad t \geq 0
$$

for any fix $\eta^{0} \in \mathbb{R}$. Similar as we have calculated for the operator $T_{2}$ on page 15 we may calculate that $T \in \mathbb{T}_{2,1,0}^{\mathrm{DAE}}$. Define

$$
T_{1}\left(y_{1}, y_{1}^{d}, y_{2}\right)(t):=\left(\begin{array}{c}
y_{1}(t) \\
y_{1}^{d}(t) \\
y_{2}(t) \\
T\left(y_{1}, y_{2}\right)(t)
\end{array}\right), \quad t \geq 0,
$$

then $T_{1} \in \mathbb{T}_{2,3,0}$, and set $T_{2}:=T$. Furthermore, define the functions

$$
\begin{aligned}
& f_{1}: \mathbb{R}^{4} \rightarrow \mathbb{R},\left(\eta_{1}, \eta_{2}, \eta_{3}, \eta_{4}\right) \mapsto-\sin \eta_{1}+\eta_{3} \eta_{2}+\eta_{3}^{2}+\eta_{2}^{2} \eta_{4}, \\
& \Gamma_{I}: \mathbb{R}^{4} \rightarrow \mathbb{R},\left(\eta_{1}, \eta_{2}, \eta_{3}, \eta_{4}\right) \mapsto \eta_{1}^{2}+\eta_{3}^{4}+1, \\
& f_{2}: \mathbb{R}^{4} \rightarrow \mathbb{R},\left(y_{1}, y_{1}^{d}, y_{2}\right) \mapsto y_{1}^{3}+y_{1}\left(y_{1}^{d}\right)^{3}+y_{2}, \\
& f_{3}: \mathbb{R} \rightarrow \mathbb{R}, \eta \mapsto \eta, \\
& \Gamma_{I I}: \mathbb{R} \rightarrow \mathbb{R}, \eta \mapsto \eta, \\
& f_{4}: \mathbb{R} \rightarrow \mathbb{R}, \eta \mapsto 1 .
\end{aligned}
$$

Then system (45) is of the form (18) with $m=2, q=1$ and $r_{1}=2$. It is straightforward to check that Assumption 3.2 is satisfied. In particular, condition (iii) is satisfied, because

$$
f_{2}^{\prime}\left(y_{1}, y_{1}^{d}, y_{2}\right)\left[\begin{array}{c}
0 \\
I_{m-q}
\end{array}\right]=\frac{\partial f_{2}}{\partial y_{2}}\left(y_{1}, y_{1}^{d}, y_{2}\right)=1
$$

is bounded. Furthermore, $f_{4}(\eta) \geq 1=: \alpha$ for all $\eta \in \mathbb{R}$, and hence we may choose $\hat{k}=2$, with which condition (24) is satisfied. 
For the simulation we choose the reference signal $y_{\mathrm{ref}}(t)=(\cos 2 t, \sin t)^{\top}$, and initial values

$$
y_{1}(0)=\dot{y}_{1}(0)=y_{2}(0)=0 \quad \text { and } \quad \eta^{0}=0 .
$$

For the controller (22) we choose the funnel functions $\varphi_{10}=\varphi_{I}=\varphi_{I I}=\varphi$ with

$$
\varphi: \mathbb{R}_{\geq 0} \rightarrow \mathbb{R}_{\geq 0}, t \mapsto \frac{1}{2} t e^{-t}+2 \arctan t
$$

It is straightforward to check that $\dot{\varphi}$ and $\ddot{\varphi}$ are bounded, thus $\varphi \in \Phi_{2}$. Moreover, since $\varphi(0)=0$, no restriction is put on the initial error and we find that 27) is satisfied and $k_{10}(0)=k_{I}(0)=1$ and $k_{I I}(0)=2$. Furthermore,

$e_{I}(0)=e_{1,1}(0)=\dot{e}_{10}(0)+k_{10}(0) e_{10}(0)=\dot{y}_{1}(0)-\dot{y}_{\text {ref }, 1}(0)+k_{10}(0)\left(y_{1}(0)-y_{\text {ref }, 1}(0)\right)=-1$, $e_{I I}(0)=y_{2}(0)-y_{\text {ref }, 2}(0)=0$,

and hence we obtain

$$
u_{I}(0)=-k_{I}(0) e_{I}(0)=1, \quad u_{I I}(0)=-k_{I I}(0) e_{I I}(0)=0 .
$$

Since $h=0$, we find that in view of Remark 4.2 the localization of $T_{2}$ satisfies $T_{2}(0,0)=0$. With this finally find that the initial value is indeed consistent, i.e., condition (26) is satisfied. We have now verified all assumptions of Theorem 4.3 by which funnel control via (22) is feasible for the system 45)

The simulation of the controller (22) applied to (45) has been performed in MATLAB (solver: ode15s, rel. tol.: $10^{-14}$, abs. tol.: $10^{-10}$ ) over the time interval $[0,10]$ and is depicted in Figure 2 ,

Figure 2 a shows the tracking error components, which stay uniformly within the funnel boundaries. The components of the generated input functions are shown in Figure $2 \mathrm{~b}$ which exhibit an acceptable performance.

\section{References}

1. Berger, T.: On differential-algebraic control systems. Ph.D. thesis, Institut für Mathematik, Technische Universität Ilmenau, Universitätsverlag Ilmenau, Germany (2014). URL http://www.db-thueringen.de/servlets/DocumentServlet?id=22652

2. Berger, T.: Zero dynamics and stabilization for linear DAEs. In: S. Schöps, A. Bartel, M. Günther, E.J.W. ter Maten, P.C. Müller (eds.) Progress in Differential-Algebraic Equations, Differential-Algebraic Equations Forum, pp. 21-45. Springer-Verlag, Berlin-Heidelberg (2014)

3. Berger, T.: Zero dynamics and funnel control of general linear differential-algebraic systems. ESAIM Control Optim. Calc. Var. 22(2), 371-403 (2016)

4. Berger, T., Ilchmann, A., Reis, T.: Zero dynamics and funnel control of linear differential-algebraic systems. Math. Control Signals Syst. 24(3), 219-263 (2012)

5. Berger, T., Ilchmann, A., Reis, T.: Funnel control for nonlinear functional differential-algebraic systems. In: Proceedings of the MTNS 2014, pp. 46-53. Groningen, NL (2014)

6. Berger, T., Ilchmann, A., Trenn, S.: The quasi-Weierstraß form for regular matrix pencils. Linear Algebra Appl. 436(10), 4052-4069 (2012). DOI 10.1016/j.laa.2009.12.036

7. Berger, T., Lê, H.H., Reis, T.: Funnel control for nonlinear systems with known strict relative degree. Automatica 87, 345-357 (2018). DOI 10.1016/j.automatica.2017.10.017

8. Berger, T., Otto, S., Reis, T., Seifried, R.: Combined open-loop and funnel control for underactuated multibody systems. Nonlinear Dynamics 95, 1977-1998 (2019). DOI 10.1007/s11071-018-4672-5

9. Berger, T., Rauert, A.L.: A universal model-free and safe adaptive cruise control mechanism. In: Proceedings of the MTNS 2018, pp. 925-932. Hong Kong (2018)

10. Berger, T., Reis, T.: Zero dynamics and funnel control for linear electrical circuits. J. Franklin Inst. 351(11), 5099-5132 (2014) 

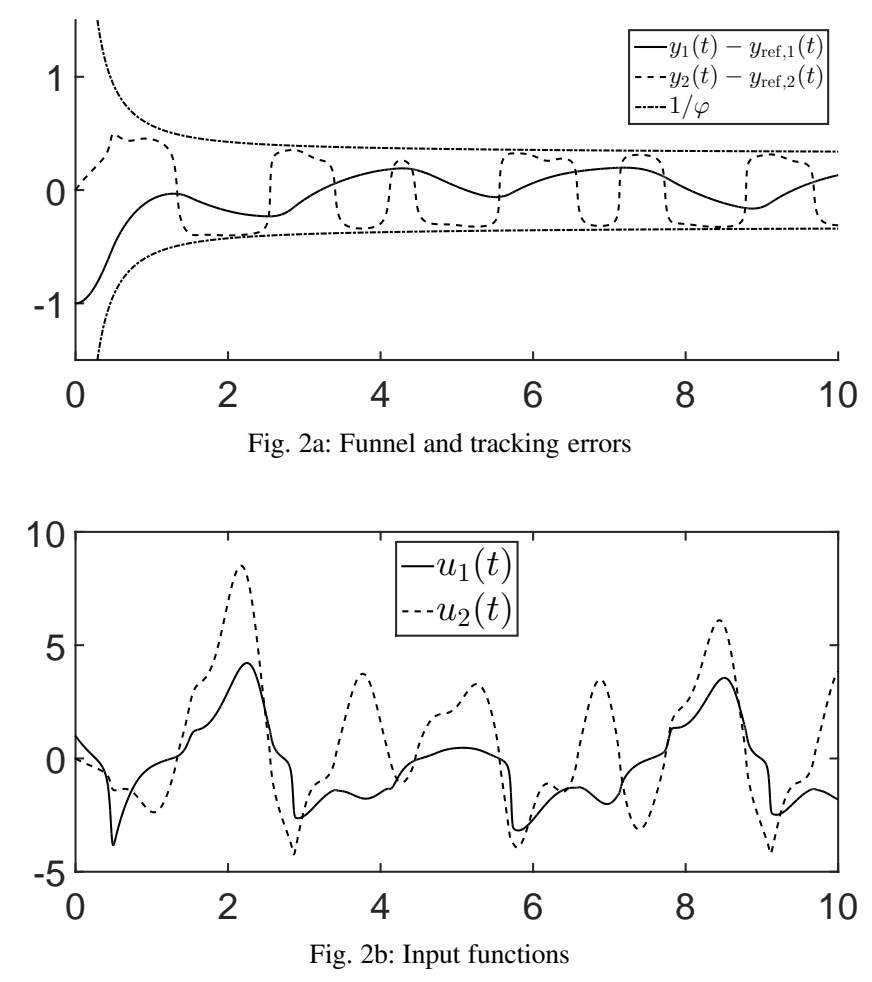

Fig. 2: Simulation of the controller (22) for the system (45).

11. Byrnes, C.I., Isidori, A.: A frequency domain philosophy for nonlinear systems, with application to stabilization and to adaptive control. In: Proc. 23rd IEEE Conf. Decis. Control, vol. 1, pp. 1569-1573 (1984)

12. Byrnes, C.I., Isidori, A.: Global feedback stabilization of nonlinear systems. In: Proc. 24th IEEE Conf. Decis. Control, Ft. Lauderdale, FL, 1, pp. 1031-1037 (1985)

13. Byrnes, C.I., Isidori, A.: Local stabilization of minimum-phase nonlinear systems. Syst. Control Lett. 11(1), 9-17 (1988)

14. Byrnes, C.I., Isidori, A.: New results and examples in nonlinear feedback stabilization. Syst. Control Lett. 12(5), 437-442 (1989)

15. Fuhrmann, P.A., Helmke, U.: The Mathematics of Networks of Linear Systems. Springer-Verlag, New York, NY (2015)

16. Hackl, C.M.: Non-identifier Based Adaptive Control in Mechatronics-Theory and Application, Lecture Notes in Control and Information Sciences, vol. 466. Springer-Verlag, Cham, Switzerland (2017)

17. Hackl, C.M., Hopfe, N., Ilchmann, A., Mueller, M., Trenn, S.: Funnel control for systems with relative degree two. SIAM J. Control Optim. 51(2), 965-995 (2013)

18. Ilchmann, A.: Decentralized tracking of interconnected systems. In: K. Hüper, J. Trumpf (eds.) Mathematical System Theory - Festschrift in Honor of Uwe Helmke on the Occasion of his Sixtieth Birthday, pp. 229-245. CreateSpace (2013)

19. Ilchmann, A., Ryan, E.P.: Universal $\lambda$-tracking for nonlinearly-perturbed systems in the presence of noise. Automatica 30(2), 337-346 (1994)

20. Ilchmann, A., Ryan, E.P.: High-gain control without identification: a survey. GAMM Mitt. 31(1), 115125 (2008)

21. Ilchmann, A., Ryan, E.P.: Performance funnels and tracking control. Int. J. Control 82(10), 1828-1840 (2009)

22. Ilchmann, A., Ryan, E.P., Sangwin, C.J.: Tracking with prescribed transient behaviour. ESAIM: Control, Optimisation and Calculus of Variations 7, 471-493 (2002) 
23. Ilchmann, A., Townley, S.B.: Simple adaptive stabilization of high-gain stabilizable systems. Syst. Control Lett. 20(3), 189-198 (1993)

24. Isidori, A.: Nonlinear Control Systems, 3rd edn. Communications and Control Engineering Series. Springer-Verlag, Berlin (1995)

25. Liberzon, D., Trenn, S.: The bang-bang funnel controller for uncertain nonlinear systems with arbitrary relative degree. IEEE Trans. Autom. Control 58(12), 3126-3141 (2013)

26. Mueller, M.: Normal form for linear systems with respect to its vector relative degree. Linear Algebra Appl. 430(4), 1292-1312 (2009)

27. Nicosia, S., Tornambè, A.: High-gain observers in the state and parameter estimation of robots having elastic joints. Syst. Control Lett. 13(4), 331-337 (1989)

28. Senfelds, A., Paugurs, A.: Electrical drive DC link power flow control with adaptive approach. In: Proc. 55th Int. Sci. Conf. Power Electr. Engg. Riga Techn. Univ., Riga, Latvia, pp. 30-33 (2014)

29. Trentelman, H.L., Stoorvogel, A.A., Hautus, M.L.J.: Control Theory for Linear Systems. Communications and Control Engineering. Springer-Verlag, London (2001). DOI 10.1007/978-1-4471-0339-4 